\title{
Mapping the thermal structure and minor species of Venus mesosphere with ALMA submillimeter observations ${ }^{\star}$
}

\author{
A. Piccialli ${ }^{\star \star}$, R. Moreno, T. Encrenaz, T. Fouchet, E. Lellouch, and T. Widemann
}

\begin{abstract}
LESIA, Observatoire de Paris, PSL Research University, CNRS, Sorbonne Universités, UPMC Univ. Paris 06, Univ. Paris Diderot, Sorbonne Paris Cité, 5 place Jules Janssen, 92195 Meudon, France

e-mail: arianna.piccialli@aeronomie.be
\end{abstract}

Received 3 April 2017 / Accepted 27 June 2017

\begin{abstract}
Context. Water vapor and sulfur compounds are key species in the photochemistry of Venus mesosphere. These species, together with mesospheric temperatures, exhibit drastic temporal variations, both on short timescales (diurnal and day-to-day) as well on long timescales, far from being understood.

Aims. We targeted $\mathrm{CO}, \mathrm{SO}, \mathrm{HDO}$ and $\mathrm{SO}_{2}$ transitions in the submillimeter range using the Atacama Large Millimeter Array (ALMA) to study their spatial and temporal variations.

Methods. Four sets of observations were acquired on different dates in November 2011 during the first ALMA Early Science observation Cycle 0 . Venus angular diameter was about $11^{\prime \prime}$ with an illumination factor of $92 \%$, so that mostly the day side of the planet was mapped. Assuming a nominal $\mathrm{CO}$ abundance profile, we retrieved vertical temperature profiles over the entire disk as a function of latitude and local time. Temperature profiles were later used to retrieve $\mathrm{SO}, \mathrm{SO}_{2}$, and $\mathrm{H}_{2} \mathrm{O}$. We used $\mathrm{HDO}$ as a tracer for water assuming a $\mathrm{D} / \mathrm{H}$ enrichment of 200 times the terrestrial value.

Results. We derived 3D maps of mesospheric temperatures in the altitude range $70-105 \mathrm{~km} . \mathrm{SO}, \mathrm{SO}_{2}$, and $\mathrm{H}_{2} \mathrm{O}$ are characterized by a negligible abundance below $\sim 85 \mathrm{~km}$ followed by an increase with altitude in the upper mesosphere. Disk-averaged SO abundances present a maximum mixing ratio of $15.0 \pm 3.1 \mathrm{ppb}$ on November 26 followed the next day by a minimum value of $9.9 \pm 1.2 \mathrm{ppb}$. Due to a very low $\mathrm{S} / \mathrm{N}, \mathrm{SO}_{2}$ could only be derived from the disk-averaged spectrum on the first day of observation revealing an abundance of $16.5 \pm 4.6 \mathrm{ppb}$. We found a $\mathrm{SO}_{2} / \mathrm{SO}$ ratio of $1.5 \pm 0.4$. Global maps of $\mathrm{SO}$ reveal strong variations both with latitude and local time and from day to day with abundance ranging from $<1$ to $15 \mathrm{ppb} . \mathrm{H}_{2} \mathrm{O}$ disk-averages retrievals reveal a steady decrease from November 14 to 27 , with the abundance varying from $3.6 \pm 0.6 \mathrm{ppm}$ on the first day to $2.9 \pm 0.7 \mathrm{ppm}$ on the last day. $\mathrm{H}_{2} \mathrm{O}$ maps reveal a slightly higher abundance on the evening side compared to the morning side and a strong depletion between the first and the second day of observation.
\end{abstract}

Key words. planets and satellites: atmospheres - planets and satellites: individual: Venus - submillimeter: planetary systems radiative transfer - instrumentation: interferometers - methods: data analysis

\section{Introduction}

Venus' mesosphere (60-120 km altitude) exhibits complex photochemistry cycles and dynamical processes that are still poorly understood. Strong variability, on both short (day-to-day) and long timescales, affects the thermal structure of this atmospheric region, especially near the morning and evening terminators, where large density and temperature variations were observed (Clancy et al. 2012; Mahieux et al. 2015; Piccialli et al. 2015). Sulfur dioxide and water vapor are key species in the photochemical cycles taking place in the troposphere and mesosphere of Venus (Mills et al. 2007; Zhang et al. 2012). Both molecules are abundant in the lower troposphere $(150 \mathrm{ppm}$ and $30 \mathrm{ppm}$ respectively, Marcq et al. 2008; Bézard et al. 2009). Convective transport, together with the Hadley circulation, carries them up to about $60 \mathrm{~km}$ where $\mathrm{SO}_{2}$ is photodissociated and combines with oxygen atoms to form $\mathrm{SO}_{3} . \mathrm{SO}_{3}$ reacts with $\mathrm{H}_{2} \mathrm{O}$ to form $\mathrm{H}_{2} \mathrm{SO}_{4}$ which condenses as clouds enshrouding the planet.

\footnotetext{
* Reduced datacubes (FITS files) are only available at the CDS via anonymous ftp to cdsarc.u-strasbg. fr (130.79.128.5) or via http://cdsarc.u-strasbg. fr/viz-bin/qcat?J/A+A/606/A53 $\star \star$ Now at the Planetary Aeronomy, Belgian Institute for Space Aeronomy, 3 av. Circulaire, 1180 Brussels, Belgium.
}

Above the clouds, both $\mathrm{SO}_{2}$ and $\mathrm{H}_{2} \mathrm{O}$ are depleted down to about $0.1-1 \mathrm{ppm}$ and $1-2 \mathrm{ppm}$ respectively.

Observations in the ultraviolet (UV) and in the infrared (IR), using Venus Express (Marcq et al. 2011; Belyaev et al. 2012), as well as in the submillimeter and in the IR, during ground-based campaigns (Sandor et al. 2010; Encrenaz et al. 2012, 2013), detected strong temporal variations of both $\mathrm{SO}$ and $\mathrm{SO}_{2}$ abundances. $\mathrm{SO}_{2}$ was found to change on a timescale as short as a couple of hours above the cloud top ( 64-70 km) (Marcq et al. 2011; Encrenaz et al. 2013, 2016). Sandor et al. (2010, 2012) observed SO to be much more depleted on the night side, consistent with the hypothesis that $\mathrm{SO}$ is a photodissociation product of $\mathrm{SO}_{2}$ with a short lifetime. Surprisingly, they also found the abundances of $\mathrm{SO}$ and $\mathrm{SO}_{2}$ at $\sim 85-100 \mathrm{~km}$ to be significantly larger than those at $70-85 \mathrm{~km}$, implying the presence of an extra source of sulfur, possibly in the form of sulfur aerosols (Sandor et al. 2012).

$\mathrm{HDO}$ and $\mathrm{H}_{2} \mathrm{O}$ have been observed in the upper mesosphere $(\sim 70-100 \mathrm{~km})$ at millimeter and submillimeter wavelengths respectively (Sandor \& Clancy 2005; Gurwell et al. 2007). These studies revealed temporal variability, with a 1-2 months timescale variations dominating. Gurwell et al. (2007) observed 
Table 1. Observations parameters.

\begin{tabular}{lccc}
\hline \hline Date (November 2011) & Time (UT) & Venus angular diameter & Beam size at $345.8 \mathrm{GHz}$ \\
\hline 14 & $22: 15-22: 53$ & $10.979^{\prime \prime}$ & $3.20^{\prime \prime} \times 2.59^{\prime \prime}$ \\
15 & $20: 39-21: 11$ & $11.007^{\prime \prime}$ & $2.74^{\prime \prime} \times 2.46^{\prime \prime}$ \\
26 & $21: 24-22: 02$ & $11.363^{\prime \prime}$ & $2.82^{\prime \prime} \times 2.42^{\prime \prime}$ \\
27 & $21: 07-21: 58$ & $11.398^{\prime \prime}$ & $2.69^{\prime \prime} \times 2.42^{\prime \prime}$ \\
\hline
\end{tabular}

a rapid decrease of water vapor over the course of five days, explained as the consequence of moderate variations in the temperature.

Ground-based observing campaigns of Venus were organized in support of space exploration observations since the early stage of Venus Express operations in 2006 (Lellouch \& Witasse 2008; Lellouch et al. 2008; Rengel et al. 2008a; Clancy et al. 2012; Sonnabend et al. 2012). Earth-based observations provide complementary information to spacecraft data by allowing a complete view of the planetary disk at a given time and a longterm coverage, which is of particular interest after the official end of Venus Express operations in January 2015. The Atacama Large Millimeter Array (ALMA) offers a unique opportunity of probing Venus upper mesosphere (60-120 km) and of monitoring minor species, winds and the thermal structure. A first set of observations was obtained in November 2011 during the first ALMA Early Science observation cycle (Encrenaz et al. 2015). These observations targeted $\mathrm{SO}_{2}, \mathrm{SO}, \mathrm{HDO}$ and $\mathrm{CO}$ transitions around $345 \mathrm{GHz}$ during four sequences of 30 minutes each. Venus angular diameter was about $11^{\prime \prime}$ with an illumination factor of $92 \%$, so that mostly the day side of the planet was mapped.

In a preliminary study, Encrenaz et al. (2015) analyzed the disk-averaged spectra for the first two runs of ALMA observations, acquired on November 14 and 15, 2011. In this paper, we present the study of the thermal structure, water vapor and sulfur species for the full ALMA data set. We analyzed all spectra maps with sufficient signal-to-noise ratio $(\mathrm{S} / \mathrm{N})$ in order to build $3 \mathrm{D}$ maps of temperature and to study the spatial and temporal variability of minor species.

ALMA observations are detailed in Sect. 2. In Sect. 3 we describe our model and the retrieval algorithm. Results are presented in Sect. 4 and discussed in Sect. 5. Our conclusions are summarized in Sect. 6.

\section{Observations and data reduction}

Observations of Venus were obtained using the ALMA interferometer within the project labeled 2011.0.00136.S. We observed - with a single receiver tuning setup - the $\mathrm{CO}, \mathrm{SO}$, $\mathrm{SO}_{2}$ and $\mathrm{HDO}$ rotational lines at frequencies of $345.795 \mathrm{GHz}$, 346.528 GHz, $346.652 \mathrm{GHz}$ and $335.395 \mathrm{GHz}$, respectively. The spectral resolution (originally $61 \mathrm{kHz}$ ) was binned to $0.35 \mathrm{MHz}$ in order to optimize the $\mathrm{S} / \mathrm{N}$. These observations were obtained on November 14, 2011, between 22:15 and 22:53 UT; on November 15, 2011, between 20:39 and 21:11 UT; on November 26, 2011, between 21:24 and 22:02 UT; and on November 27, 2011, between 21:07 and 21:58 UT. During each observing run, between 14 and 17 antennas of the main ALMA interferometer array were available. The planet's apparent angular diameter varied between $10.97^{\prime \prime}$ to $11.39^{\prime \prime}$, which we finally re-scaled to a common scale of $11^{\prime \prime}$ for a better day-to-day comparison. Table 1 summarizes all observation parameters. The average illumination factor was of 0.92 , therefore we observed mainly in the day side. A small part of the night side is also visible on the left-side of the maps presented in Figs. 1-4 beyond the evening terminator in the east limb.

The data reduction was performed with the ALMA Common Astronomy Software Applications package $\left(\mathrm{CASA}^{1}\right)$. In order to calibrate the raw visibilities (i.e., the Fourier transform of the image that measures an interferometer array), as a first step we calibrated the spectral response of the heterodyne receiver bandwidth (bandpass) on the strong quasar J1924-292. To reduce the effect of atmospheric turbulence in front of the antenna, the amplitude and phase variation versus time were calibrated using the quasar NRAO530 (measured every eight minutes) which was nearby Venus. The planet topocentric velocity was corrected directly on the individual visibility using the task CVEL in order to obtain spectra in the planetocentric velocity frame. The next steps were performed using the GILDAS software $^{2}$, a radio-astronomical package developed by the Institut de Radio-Astronomie Millimétrique (IRAM). To improve the phase calibration and therefore the images quality, a "self-calibration" task was applied to the phase of all visibilities using the known continuum disk-like shape of Venus. Moreover, the measured fluxes were scaled to the continuum of our Venus model. The relative calibration is mainly measured with the day-to-day continuum flux variation of the deconvolved images. Such a method gives a relative flux accuracy of $1-2 \%$, allowing an accurate comparison for the short/long term variations of the ALMA data analysis. On the other hand, our ALMA absolute flux accuracy of Venus depends on the continuum model uncertainty which is of $5 \%$. This value is driven by the absolute uncertainty of the Venus atmospheric temperature at an altitude of $57 \mathrm{~km}$.

For clarity, we have oriented the North Pole on the top of all of our figures, by correcting the planet's North Pole position angle directly in all visibilities. Since the synthesized beams were different on each day, due to a different number of antenna and maximum baseline, we had to perform a baseline tapering to obtain a similar synthesized beam for the four observing runs. The elliptical tapered synthesized beams at $345.8 \mathrm{GHz}$ are summarized in Table 1 . The final step corresponds to the imaging process, in which we have obtained the deconvolved images using a CLEAN task with the Hogbom algorithm.

$\mathrm{CO}$ and $\mathrm{SO}$ absorption lines were detected and mapped on each day (peak $\mathrm{S} / \mathrm{N}$ of the spectral lines maps on $\mathrm{CO}>1000$, and on $\mathrm{SO}>10$ ), but HDO absorption line was only detected on November 14, 15, and 27, 2011 and $\mathrm{SO}_{2}$ absorption line was only detected on November 14, 2011. Moreover, HDO had a high enough $\mathrm{S} / \mathrm{N}$ to be mapped only on the first two runs (peak $\mathrm{S} / \mathrm{N}$ of the spectral lines maps on HDO $>6$ ). The analysis of the spectral maps in Sect. 3 are therefore focused on the spectral maps detections with sufficient $\mathrm{S} / \mathrm{N}$ : $\mathrm{CO}$ and $\mathrm{SO}$ on all observed runs, and HDO on November 14 and 15, 2011. Figures 1-4 show the final spectral maps of the $\mathrm{CO}$ and SO obtained on November 27, 2011 and HDO on November 15, 2011. Most of the other observations used in the spectral maps analysis in Sect. 3 were

\footnotetext{
1 https://casa.nrao.edu/

2 https://WWW.iram. fr/IRAMFR/GILDAS/
} 

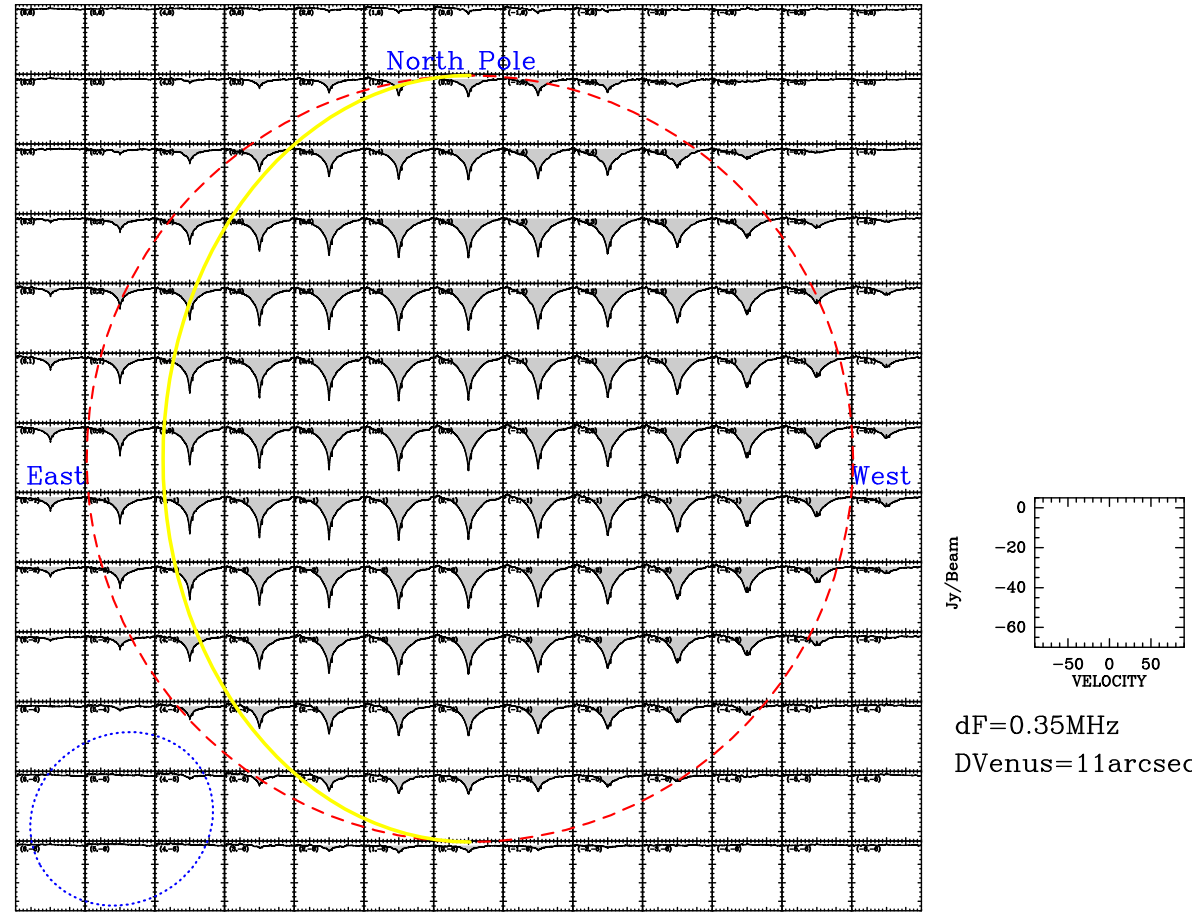

$\mathrm{dF}=0.35 \mathrm{MHz}$

DVenus $=11$ arcsec
Fig. 1. Map of the $\mathrm{CO}(3-2)$ transition on Venus at $345.795 \mathrm{GHz}$ observed on November 27, 2011. The spectral resolution is $0.35 \mathrm{MHz}$ and the bandwidth is $150 \mathrm{MHz}$. The planet's angular diameter is $11^{\prime \prime}$ and is shown by a red circle. The evening terminator is indicated with a yellow line. The synthesized beam is indicated with a blue ellipse (in the lower left corner) of $2.69^{\prime \prime} \times 2.42^{\prime \prime}$

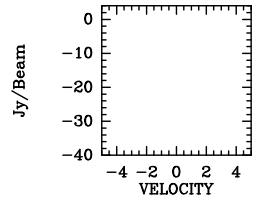

$\mathrm{dF}=0.35 \mathrm{MHz}$

DVenus $=11$ arcsec

Fig. 2. Map of the $\mathrm{CO}(3-2)$ transition in the central core $( \pm 5 \mathrm{MHz})$ of the line on Venus at 345.795 GHz observed on November 27, 2011 (i.e., magnification of Fig. 1).

presented in the Encrenaz et al. (2015) paper which focused on the disk-averaged analysis and disk-center spectra.

\section{Data analysis}

\subsection{Radiative transfer model}

We built a line-by-line non-scattering radiative transfer model adapted from Encrenaz et al. (2015) to compute synthetic spectra of Venus in the submillimeter range. Our model atmosphere consists of 141 levels equally spaced ranging from 0 to $140 \mathrm{~km}$. As in Encrenaz et al. (2015), spectroscopic parameters were taken from the JPL Molecular Spectroscopy catalog
(Poynter \& Pickett 1985). The values used for the computations are summarized in Table 2. The parameter $v_{0}$ is the central frequency of the line, $I_{0}$ is the line intensity at $T=300 \mathrm{~K}$, and $E_{0}$ is the energy level of the lower state of the transition (Poynter \& Pickett 1985; Pickett et al. 1998). $\Delta \gamma$ is the HWHM (Half-width half-maximum) of the pressure broadening at 1 bar and $T=296 \mathrm{~K}$ taken from the HITRAN database (Rothman et al. 2005). A Lorentzian profile is used to calculate the opacity. A discussion about the validity of the broadening factor and its impact on the results can be found in Encrenaz et al. (2015). Finally, $n$ is the coefficient of temperature dependence of the broadening factor $\Delta \gamma\left(T^{n}\right)$ obtained respectively by Varanasi et al. (1987) for CO, by Varanasi (1971) 

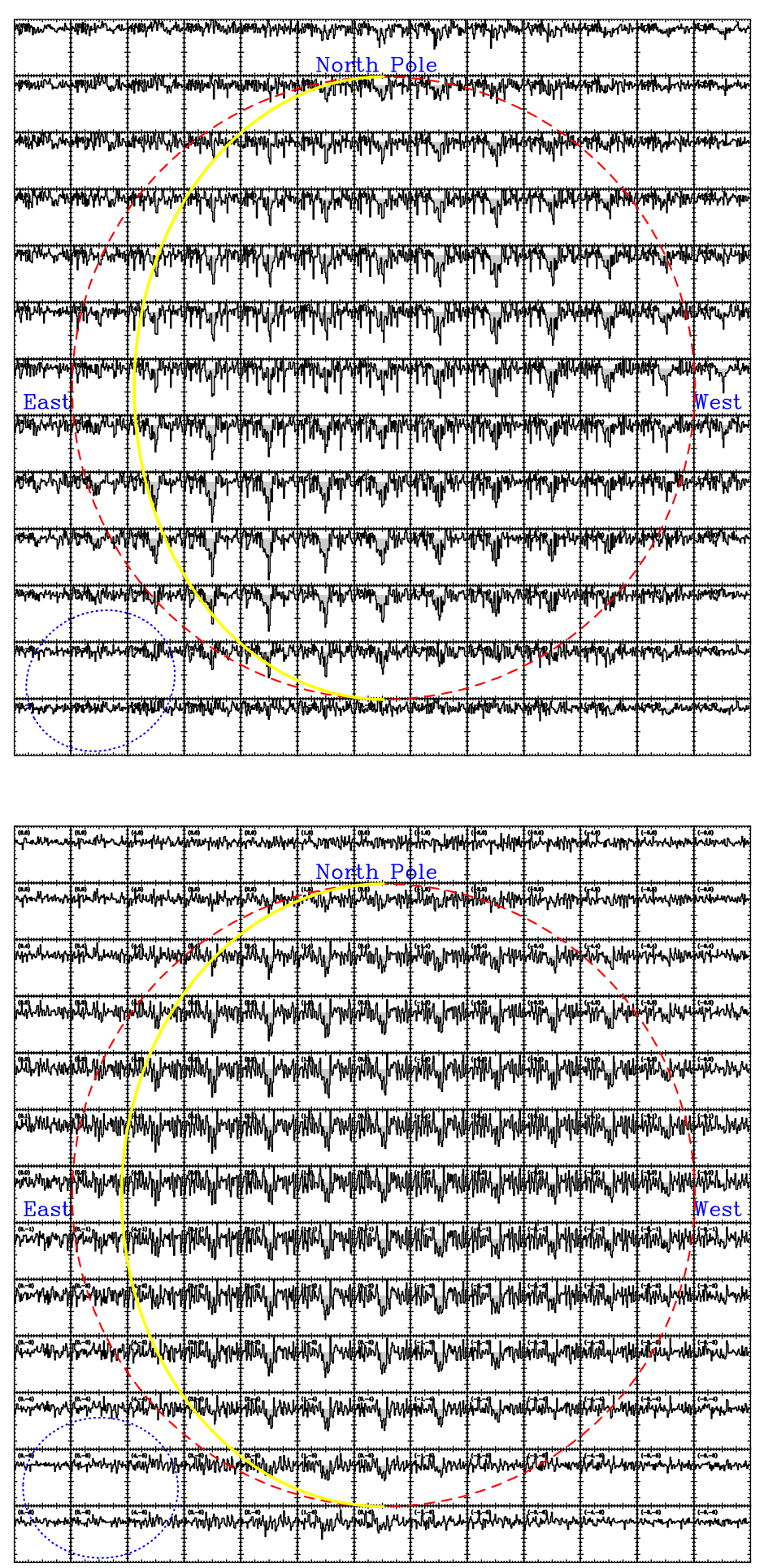

for HDO, and by Bézard et al. (1990) for SO and $\mathrm{SO}_{2}$. At millimeter wavelengths the continuum opacity is mainly defined by the $\mathrm{CO}_{2}-\mathrm{CO}_{2}$ collision induced absorption. The $\mathrm{CO}_{2}$ absorption coefficient was then calculated following the method of Ho et al. (1966). Our radiative transfer algorithm takes into account the contributions of the individual line-of-sight calculated as a function of the distance $D$ from Venus's center (with $D$ in the range $0-6051.5 \mathrm{~km}$ ), then convolved with the synthetic beam of the array and finally co-added. The limb contribution was not included. Synthetic spectra were computed with a sampling of $0.1 \mathrm{MHz}$ before convolution to the appropriate instrumental spectral response function $(\Delta v=0.35 \mathrm{MHz})$.

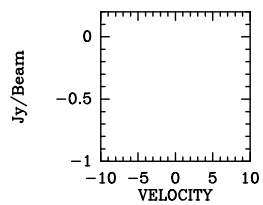

$\mathrm{dF}=0.35 \mathrm{MHz}$

DVenus $=11$ arcsec
Fig. 3. Map of the SO line on Venus at 346.528 GHz observed on November 27, 2011. The spectral resolution is $0.35 \mathrm{MHz}$. The planet's angular diameter is $11^{\prime \prime}$ and is shown by a red circle. The evening terminator is indicated with a yellow line. The synthesized beam is indicated with a blue ellipse (in the lower left corner) of $2.68^{\prime \prime} \times 2.41^{\prime \prime}$.

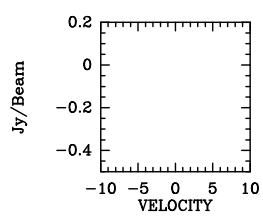

$\mathrm{dF}=0.35 \mathrm{MHz}$ DVenus $=11$ arcsec
Fig. 4. Map of the HDO line on Venus at $335.395 \mathrm{GHz}$ observed on November 15 , 2011. The spectral resolution is $0.35 \mathrm{MHz}$. The planet's angular diameter is $11^{\prime \prime}$ and is shown by a red circle. The evening terminator is indicated with a yellow line. The synthesized beam is indicated with a blue ellipse (in the lower left corner) of $2.65^{\prime \prime} \times 2.38^{\prime \prime}$.

\subsection{Temperature retrieval}

The CO line contrast and shape depend both on the atmospheric temperature profile and on the $\mathrm{CO}$ mixing ratio profile. Our dataset contains only one CO transition $\left(v_{0}=345.7960 \mathrm{GHz}\right)$, so it is not possible to retrieve the $\mathrm{CO}$ mixing ratio and the temperature simultaneously. In order to retrieve vertical temperature profiles, we assumed a nominal day side $\mathrm{CO}$ abundance profile (Fig. 5) obtained as an average of the 2000-2002 (Fig. 5) and 2007-2009 (Fig. 5) CO mixing ratio profiles from Clancy et al. (2012) in the altitude range 70-106 km. We fixed a CO mixing ratio of $27 \mathrm{ppm}$ at $36 \mathrm{~km}$ (Marcq et al. 2008) and 
Table 2. Spectroscopic parameters of the observed $\mathrm{CO}, \mathrm{HDO}, \mathrm{SO}$ and $\mathrm{SO}_{2}$ lines.

\begin{tabular}{lcccc}
\hline \hline Parameters & $\mathrm{CO}$ & $\mathrm{HDO}$ & $\mathrm{SO}$ & $\mathrm{SO}_{2}$ \\
\hline$v_{0}(\mathrm{GHz})$ & 345.7960 & 335.3955 & 346.5285 & 346.652 \\
$I_{0}\left(\mathrm{~cm}^{-2} \mathrm{~atm}^{-1}\right)$ & $0.8150 \times 10^{-22}$ & $9.0506 \times 10^{-23}$ & $5.3368 \times 10^{-21}$ & $1.1060 \times 10^{-21}$ \\
$E_{0}\left(\mathrm{~cm}^{-1}\right)$ & 11.5350 & 221.8395 & 43.1928 & 105.2994 \\
$\Delta \gamma\left(\mathrm{cm}^{-1} \mathrm{~atm}^{-1}\right)$ & 0.09 & 0.15 & 0.09 & 0.15 \\
$n$ & -0.75 & -0.35 & -0.75 & -0.75 \\
\hline
\end{tabular}

Notes. See text for detail.

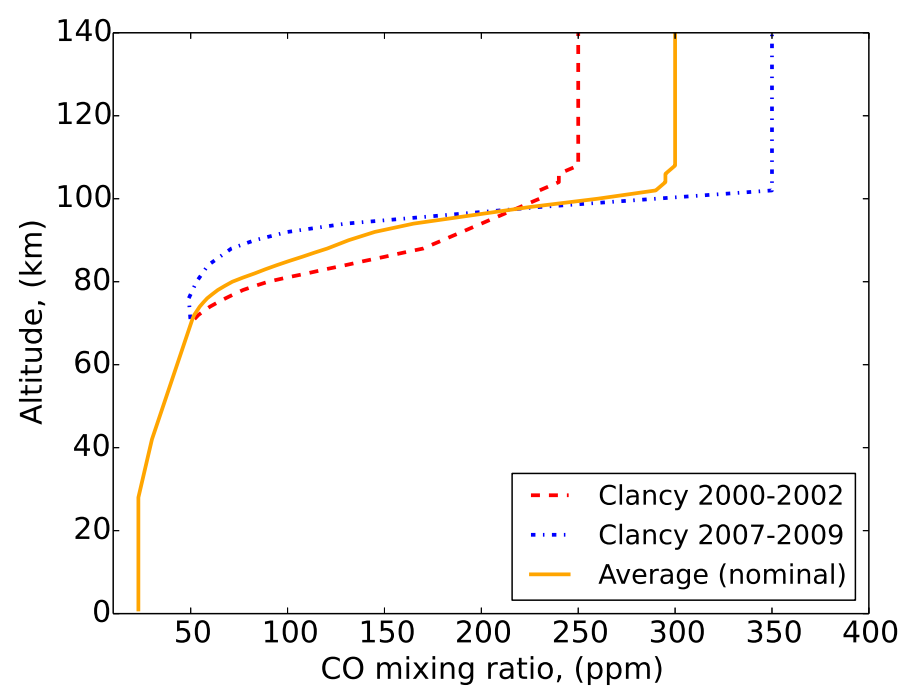

Fig. 5. CO vertical profile used for the temperature retrieval (orange line). CO mixing ratios profiles in the altitude range $70-106 \mathrm{~km}$ were obtained respectively from 2000-2002 (red dashed line) and 2007-2009 (blue dash-dot line) observations (Clancy et al. 2012). Above $106 \mathrm{~km}$ we assumed a constant value.

30 ppm at $42 \mathrm{~km}$ (Bézard \& de Bergh 2007). We assumed a constant value above $106 \mathrm{~km}$ and below $28 \mathrm{~km}$ with a linear increase of $\mathrm{CO}$ mixing ratio between 42 and $70 \mathrm{~km}$, as seen in Bézard \& de Bergh (2007).

We tested the sensitivity of the retrieved temperature profiles to the initial choice of the $\mathrm{CO}$ mixing ratio profile. We used the day side CO abundance profiles 2007-2009 (Fig. 5) and 2000-2002 (Fig. 5) to retrieve the temperature profile in five regions. We note that the CO abundance 2007-2009 profile is about two times higher than the 2000-2002 profile in the altitude range $70-90 \mathrm{~km}$. We found a percentage change in the temperature profiles obtained using the two initial $\mathrm{CO}$ abundance profiles smaller than $5 \%( \pm 10 \mathrm{~K})$ at $85 \mathrm{~km}$ and smaller than $2 \%$ $( \pm 4 \mathrm{~K})$ above $90 \mathrm{~km}$.

\section{Retrieval method}

To retrieve the temperature, we adapted for Venus' atmosphere a constrained and regularized retrieval algorithm detailed in Conrath et al. (1998) and more recently applied in Lellouch et al. (2017) and Fouchet et al. (2016). An a priori temperature profile is required as an initial input, temperature profiles are then constrained to stay close to this a priori at altitudes where the measurements contain no information. At altitudes where information is available, the departure from the a priori profile was regularized, or smoothed, to inhibit spurious vertical oscillations.
The radiance can be linearized with the temperature as:

$\Delta I_{i}=\sum_{j=1}^{n} \frac{\partial I_{i}}{\partial T_{j}} \Delta T_{j}$,

where $\Delta I_{i}$ is the difference between the observed and the synthetic radiance at frequency $v_{i}$, and $\Delta T_{j}$ is the difference between the actual value of the temperature and its a priori value used to calculate the synthetic spectrum. This equation can be written in vectorial form as

$\Delta I=K \Delta T$.

Here $K$ is the Jacobian matrix (the matrix of the functional derivatives) defined as: $K_{i j}=\partial I_{i} / \partial T_{j}$, where $I_{i}$ is the radiance at the wavelength $v_{i}$ and $T_{j}$ is the temperature at the altitude $z_{j}$. A formal solution of this equation is

$\boldsymbol{\Delta} \boldsymbol{T}=U \boldsymbol{\Delta} \boldsymbol{I} \quad$ with $\quad U=\alpha S K^{T}\left(\alpha K S K^{T}+E\right)^{-1}$.

In this equation, $S$ is the correlation matrix used to smooth the output vertical profile; $\alpha$ is a factor that determines the weight assigned to the data with respect to the a priori profile; $E$ is the covariance matrix of the measurement. In our cases it reduces to a diagonal matrix. The values of $S$ and $\alpha$ were determined through numerical experiment. The retrieval algorithm proceeds by successive iterations. In practice, the vector $\Delta T$ is the output of the inversion process that must be added to the a priori temperature profile for the next iteration. At each iteration we estimated the fit quality as: $\chi^{2}=\sum_{i}\left(\frac{\Delta I_{i}}{E_{i}}\right)^{2}$. The iteration process was stopped when the $\chi^{2}$ changed by less than $1 \%$ from one iteration to another.

In order to fit the wings of the CO line and the center of the line we needed to separate the iteration process in two steps. In a first step, we performed the temperature inversion over the entire CO band $(\Delta v=200 \mathrm{MHz})$. This first retrieval allowed us to obtain a good fit of the wings of the $\mathrm{CO}$ band, but not of the center of the line. In the second inversion, only the center of the CO line $(\Delta v=4.5 \mathrm{MHz})$ was taken in consideration. Figure 6 displays, as an example, on the left panel the CO ALMA spectrum for observation \#313 at the disk center on November 14, 2011 , and on the right panel the retrieved temperature profile. It is compared to VIRA (Venus International Reference Atmosphere model) temperature profile, used as a priori.

As explained in Guerlet et al. (2009), the information content of the data (the altitudes sounded and the vertical resolution) is given by the functional derivatives $\partial I_{i} / \partial T_{j}$. Figure 7 shows the vertical profiles of the functional derivative for the observation at the disk center \#313 on November 14, 2011 at the center of the line $v_{i}=345.796 \mathrm{GHz}$, and at the frequencies: $\Delta v=1 \mathrm{MHz}$; $\Delta v=10 \mathrm{MHz} ; \Delta v=500 \mathrm{MHz}$. The vertical profiles of the temperature functional derivatives show where most of the radiation at each wavelength originates from. The $\mathrm{CO}$ line gives us information on the temperature at different altitudes in the range 
Spectrum CO \#313; Coordinates: (0"; 0")
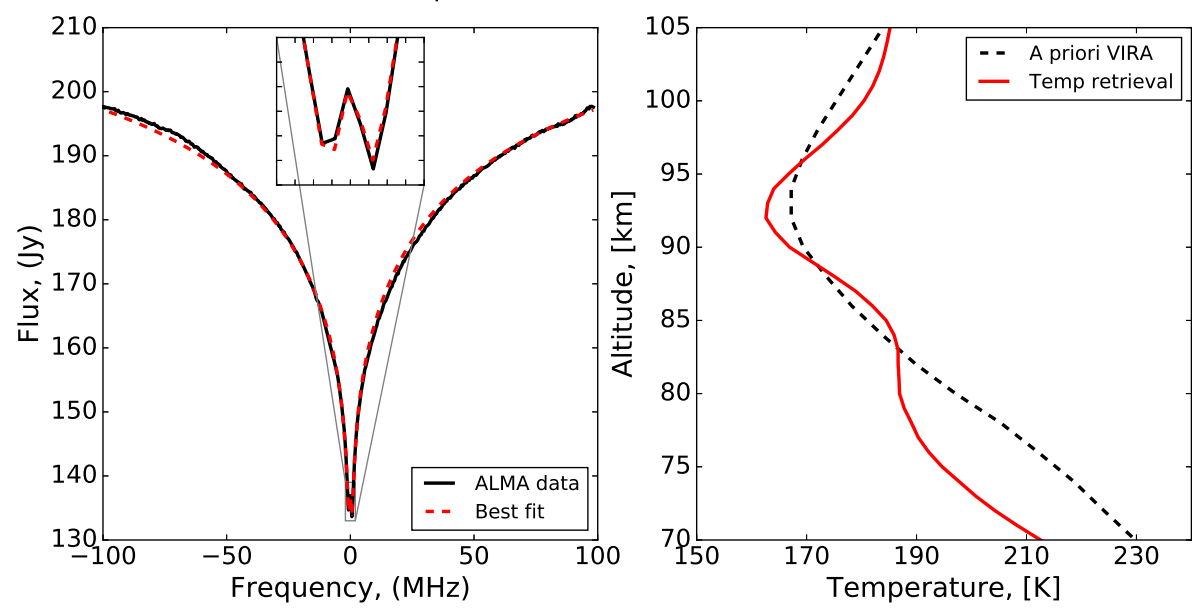

Fig. 6. Left: CO ALMA spectrum at $345.7960 \mathrm{GHz}$ for observation \#313 at the disk center (black solid line) on November 14, 2011. We over-plotted a synthetic spectrum (red dotted line) obtained using the nominal $\mathrm{CO}$ profile of Fig. 5 (orange line) and the retrieved temperature profile (red solid line) on the right panel. Right: Retrieved temperature profile (red solid line) as function of altitude. VIRA day side temperature profile (black dotted line) used as a priori is also displayed.

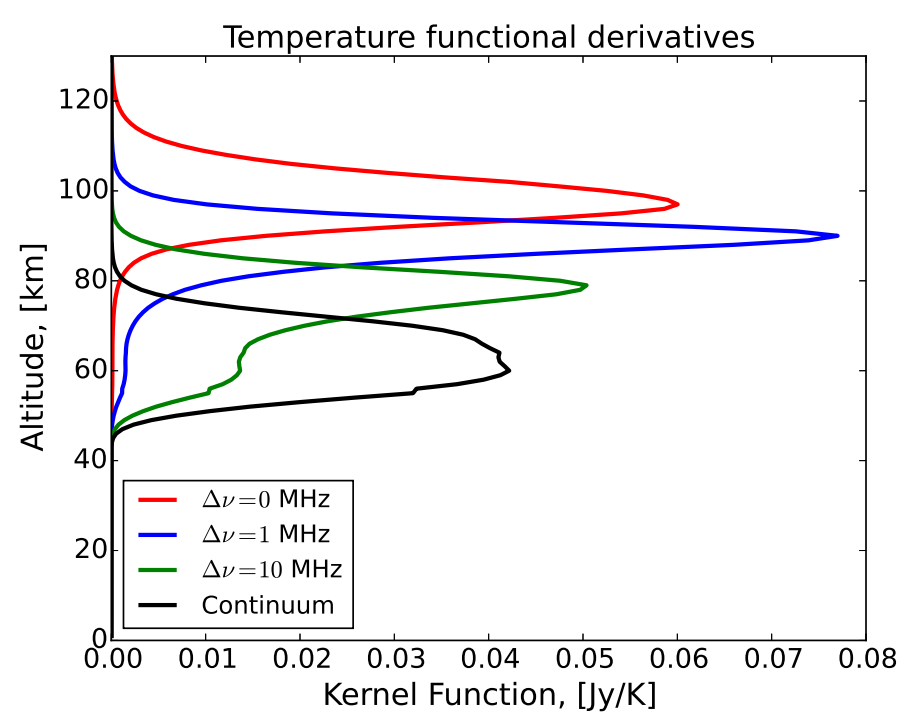

Fig. 7. Functional derivative as function of altitude for observation \#313 on November 14, 2011 at frequencies: $v_{i}=345.796 \mathrm{GHz}$ (red line); $v_{i}=345.797 \mathrm{GHz}$ (blue line); $v_{i}=345.806 \mathrm{GHz}$ (green line); $v_{i}=$ $347.296 \mathrm{GHz}$ (black line).

$60-120 \mathrm{~km}$. The center of the CO line at $v_{i}=345.796 \mathrm{GHz}$ probes the altitude region around $100 \mathrm{~km}$. As frequency changes from the line center to the wings, the contribution to temperature from the lower mesosphere increases and the continuum has a maximum of sensitivity around $60-70 \mathrm{~km}$. It is however difficult to estimate accurately the altitude where temperature retrievals start to be independent from the continuum model. Therefore we have conservatively estimated the minimum altitude of the temperature retrievals at $70 \mathrm{~km}$, in agreement with Clancy et al. (2012).

The choice of the a priori profile plays an important role in the retrieval process. We assumed as an a priori temperature profile the VIRA day side profile (latitude: $0-30^{\circ}$ ) from Seiff et al. (1985) from the surface up to an altitude of $100 \mathrm{~km}$; above this altitude we assumed a linear profile up to $140 \mathrm{~km}$. We tested the dependency of temperature retrievals to the choice of the a priori profile. Therefore, we used a "warm" and a "cold" profile as a priori to retrieve the temperature for observation \#313 in the center of Venus' disk. As can be seen in Fig. 8, the warm and cold a priori profiles coincide with VIRA below $60 \mathrm{~km}$ of altitude, above this altitude they diverge. The retrieved temperature

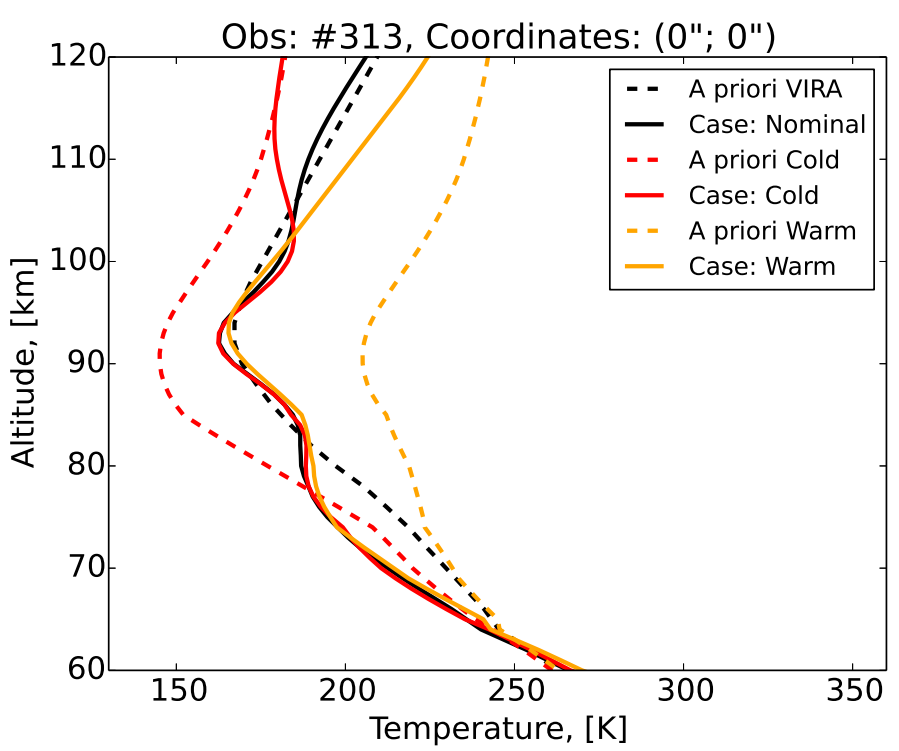

Fig. 8. Retrieved temperature profiles (solid lines), starting from three different first-guess profiles (dashed lines), for the disk center observation \#313 on November, 14. We used the CO vertical profile (orange line) corresponding to the nominal case of Fig. 5.

profiles for the three cases converge below $\sim 103 \mathrm{~km}$ of altitude within $3 \%( \pm 6 \mathrm{~K})$ of percentage change. Above this altitude, retrieved temperature profiles depend on the choice of the a priori.

\subsection{Retrieval of minor species}

The retrieved temperature profiles were later used to model the spectra of minor species. As for temperature retrievals, we followed the same method of Conrath et al. (1998). The radiance can be linearized as

$\Delta I_{i}=\sum_{j=1}^{n} \frac{\partial I_{i}}{\partial \ln \left(q_{j}\right)} \Delta \ln \left(q_{j}\right)$,

where $q$ is the abundance profile. In vectorial form this equation becomes: $\Delta I=M \Delta \ln (q)$, where $M$ is the functional derivative matrix with respect to the logarithm of the mixing ratio of each molecule. To calculate $M$, we simply calculated two spectra with mixing ratios $q$ differing by $10 \%$, from which we could derive $\mathrm{d} I_{i} / \mathrm{d} \ln (q)$. We started from an a priori $q$ profile and each iteration gave as output the vector $\Delta \ln (q)$ that needed to be added 
to the a priori profile for the next iteration. The iteration process continued until the change in $\chi^{2}$ was less than $1 \%$.

\subsubsection{SO}

Since 2004, Sandor et al. (2010) have monitored the SO transition revealing that it strongly varies with time. As an a priori profile for our retrieval, we therefore chose two extreme cases to allow all possibilities (See Fig. 9). We performed two inversions, starting respectively from a depleted and a rich a priori constant SO profile. As can be seen in Fig. 9 (bottom-left), the two retrieved profiles have a similar behavior between about 60 and $92 \mathrm{~km}$ of altitude and they both fit the SO spectrum (Fig. 9, top). By calculating the functional derivatives for molecular abundances, we can understand at which pressure level (altitude) measurements are sensitive to the molecular abundance. Analyzing the functional derivatives (kernel functions) for SO (Fig. 9 bottom-right), it is clear that the absorption spectra are sensitive to altitudes $80-100 \mathrm{~km}$ and have a peak sensitivity around $92 \mathrm{~km}$. Measurements present a much weaker sensitivity to the molecular abundance below $80 \mathrm{~km}$. Above $90 \mathrm{~km}$, since a unique solution cannot be found, we assumed a constant value, which is given by the intersection of the two curves and that also fits the observed spectrum. It is then possible to derive a column density of SO and an approximate constant profile above $90 \mathrm{~km}$, which can be used for comparison with previous literature observations.

We tested the sensitivity of SO retrievals to different temperature profiles. We used as temperature profiles those derived by assuming respectively the day side $\mathrm{CO}$ abundance profile of 2007-2009 (Fig. 5) and 2000-2002 (Fig. 5). The percentage change of the retrieved SO column densities varies from one observation to another and it is in the range $1-6 \%$.

\subsubsection{Water}

Following the same method described by Encrenaz et al. (2015), we used HDO as a tracer for water. For consistency with that study, we assumed a D/H enrichment of 200 times the terrestrial value, which is also convenient for comparison with previous studies.

For the retrieval of $\mathrm{H}_{2} \mathrm{O}$ we proceeded in a similar way as for SO. We performed two inversions, starting respectively from a depleted and a rich a priori constant $\mathrm{H}_{2} \mathrm{O}$ profile. The two retrieved $\mathrm{H}_{2} \mathrm{O}$ profiles present a similar behavior between 60-90 km and they both fit the HDO spectrum (Fig. 10, top). Above $90 \mathrm{~km}$, since a unique solution cannot be found, we assumed a constant value, which is given by the intersection of the two curves. The kernel functions peak at about $94 \mathrm{~km}$; the maximum of information comes from $\sim 80-100 \mathrm{~km}$. Measurements present a much weaker sensitivity to the molecular abundance below $80 \mathrm{~km}$.

We also tested the sensitivity of $\mathrm{H}_{2} \mathrm{O}$ to different temperature retrievals (and $\mathrm{CO}$ input vertical profiles). We proceeded as in the case of SO: we used as temperature profiles those derived by assuming respectively the day side $\mathrm{CO}$ abundance profile of 2007-2009 (Fig. 5) and 2000-2002 (Fig. 5). The percentage change of the $\mathrm{H}_{2} \mathrm{O}$ column densities varies between $2.5-5 \%$ depending on the observation.

\subsection{Error analysis}

Table 3 summarizes the sources of error and the sensitivity of the retrieved temperature and minor species to them. Errors on the
Table 3. Uncertainties in the retrieval of temperature (T), SO, HDO and $\mathrm{SO}_{2}$ abundance.

\begin{tabular}{rcccc}
\hline \hline Error source & $\delta T$ & $\delta \mathrm{SO}$ & $\delta \mathrm{H}_{2} \mathrm{O}$ & $\delta \mathrm{SO}_{2}$ \\
\hline rms/line flux & & & & \\
14 Nov. & & $\pm 5.6 \%$ & $\pm 14 \%$ & $\pm 24 \%$ \\
15 Nov. & & $\pm 6.4 \%$ & $\pm 15 \%$ & - \\
26 Nov. & & $\pm 18 \%$ & - & - \\
27 Nov. & & $\pm 8.9 \%$ & $\pm 22 \%$ & - \\
\hline CO input & $\pm 5 \%$ & $\pm 1-6 \%$ & $\pm 2.5-5 \%$ & $\pm 3-5 \%$ \\
A priori $T$ & $\pm 3 \%$ & - & - & - \\
\hline Total error & & & & \\
14 Nov. & $\pm 8 \%$ & $\pm 9 \%$ & $\pm 18 \%$ & $\pm 28 \%$ \\
15 Nov. & $\pm 8 \%$ & $\pm 10 \%$ & $\pm 19 \%$ & - \\
26 Nov. & $\pm 8 \%$ & $\pm 21 \%$ & - & - \\
27 Nov. & $\pm 8 \%$ & $\pm 12 \%$ & $\pm 26 \%$ & - \\
\hline
\end{tabular}

retrieved temperature and on minor species abundances result from random noise in the measurements and they are quantified in the inversion method (see Conrath et al. 1998). These random errors are for temperature $<0.1 \mathrm{~K}$. However, uncertainties are mainly dominated by a priori errors. Temperatures are particularly stable against a change in the a priori profile between 70 and $103 \mathrm{~km}$ which induces a $\pm 3 \%( \pm 6 \mathrm{~K}$ ) uncertainty (Fig. 8). Another source of error on the retrieval of temperature profiles comes from the $\mathrm{CO}$ profile. A change in the input $\mathrm{CO}$ abundance profile (we used the 2000-2002 and 2007-2009 CO abundance profiles as tests) produces a $\pm 5 \%( \pm 10 \mathrm{~K}$ at $85 \mathrm{~km})$ uncertainty on temperature. The choice of the $\mathrm{CO}$ input profile has an effect also on the retrieval of minor species. The $\mathrm{CO}$ uncertainty induces respectively an error on the column density: of 1-6\% on $\mathrm{SO}$, and of $2.5-5 \%$ on $\mathrm{H}_{2} \mathrm{O}$. As detailed in Sect. 2, the relative calibration uncertainty is about $1-2 \%$. This allows an accurate analysis of the day-to-day variability. The global uncertainties (linearly-summed) are listed in Table 3.

\section{Results}

\subsection{Temporal variations of disk averages}

As first step, we applied the inversion algorithm to spectra integrated over the entire disk. Figure 11 displays the results for temperature profiles. Vertical temperature profiles were retrieved for the four runs of observations and compared to the a priori VIRA temperature profile. The uncertainty of the November 14 temperature profile is represented as an example by the colored area. All profiles are colder than VIRA, they present a similar behavior with a steady decrease up to about $85 \mathrm{~km}$, followed by an increase of temperature above $100 \mathrm{~km}$. Day-to-day variations are observed with a maximum departure of $\sim 10 \mathrm{~K}$ from the VIRA profile observed on November 15 . Such variations lay well within uncertainties on temperature. Moreover, temporal variations of $10-15 \mathrm{~K}$ in the upper mesosphere were also observed previously on both day- and night sides on timescales as short as $24 \mathrm{~h}$ by Clancy et al. (2003). With the exception of the November 15 profile, all profiles converge above $100 \mathrm{~km}$.

The retrieved temperature profiles were later used to derive the minor components: $\mathrm{SO}, \mathrm{H}_{2} \mathrm{O}$ and $\mathrm{SO}_{2}$. As explained in more detail earlier, we used HDO as a tracer for water. Due to a low $\mathrm{S} / \mathrm{N}, \mathrm{H}_{2} \mathrm{O}$ and $\mathrm{SO}_{2}$ could not be retrieved for all four sets of observation. Table 4 summarizes the results of the retrievals. SO does not change much from one day to another, with the exception of November 26: we observe first a maximum mixing 

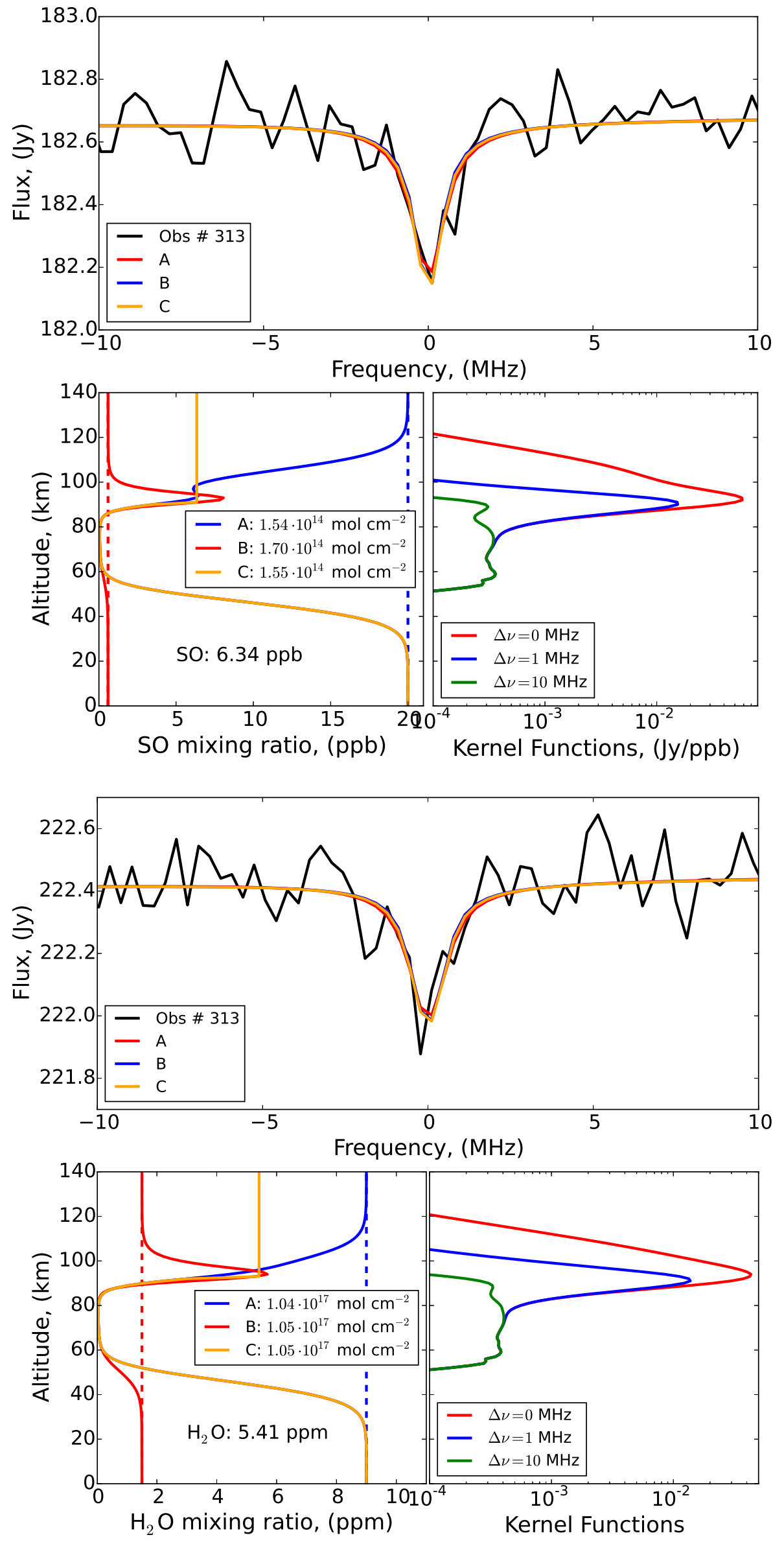

Fig. 9. Top: SO spectrum at $346.528 \mathrm{GHz}$ for observation \#313 at the disk center on November 15, 2011 (black solid line). Bottom, left: SO profile obtained from the depleted firstguess profile (red). SO profile obtained from the rich first-guess profile (blue). Final SO profile obtained from the intersection of the first two above $\sim 90 \mathrm{~km}$ (orange). Dashed lines are respectively the depleted (red) and the rich (blue) a priori profiles. Bottom, right: Functional derivatives as function of altitude for observation \#313 on November 15, 2011 at frequencies: $v_{i}=346.528 \mathrm{GHz}$ (red line); $v_{i}=$ $346.529 \mathrm{GHz}$ (blue line); $v_{i}=346.538 \mathrm{GHz}$ (green line).

Fig. 10. Top: $\mathrm{HDO}$ spectrum at $335.3955 \mathrm{GHz}$ for observation \#313 at the disk center on November 15, 2011 (black solid line). Bottom, left: $\mathrm{H}_{2} \mathrm{O}$ profile obtained from the depleted first-guess profile (red). $\mathrm{H}_{2} \mathrm{O}$ profile obtained from the rich first-guess profile (blue). Final $\mathrm{H}_{2} \mathrm{O}$ profile obtained from the intersection of the first two above $\sim 90 \mathrm{~km}$ (orange). Dashed lines are respectively the depleted (red) and the rich (blue) a priori profiles. Bottom, right: Functional derivatives as function of altitude for observation \#313 on November 15, 2011 at frequencies: $v_{i}=346.528 \mathrm{GHz}$ (red line); $v_{i}=$ $346.529 \mathrm{GHz}$ (blue line); $v_{i}=346.538 \mathrm{GHz}$ (green line). 
Table 4. Retrieved disk-averaged abundance for $\mathrm{SO}, \mathrm{H}_{2} \mathrm{O}$ and $\mathrm{SO}_{2}$.

\begin{tabular}{lccc}
\hline \hline Day & $\mathrm{SO}$ & $\mathrm{H}_{2} \mathrm{O}$ & $\mathrm{SO}_{2}$ \\
\hline \multirow{2}{*}{ 14th Nov. } & $11.2 \pm 1.0 \mathrm{ppb}$ & $3.6 \pm 0.6 \mathrm{ppm}$ & $16.5 \pm 4.6 \mathrm{ppb}$ \\
& $(3.3 \pm 0.3) \times 10^{14} \mathrm{~mol} \mathrm{~cm}^{-2}$ & $(1.2 \pm 0.2) \times 10^{17} \mathrm{~mol} \mathrm{~cm}^{-2}$ & $(4.9 \pm 1.4) \times 10^{14} \mathrm{~mol} \mathrm{~cm}^{-2}$ \\
\hline \multirow{2}{*}{ 15th Nov. } & $10.5 \pm 1.0 \mathrm{ppb}$ & $3.3 \pm 0.6 \mathrm{ppm}$ & - \\
& $(3.1 \pm 0.3) \times 10^{14} \mathrm{~mol} \mathrm{~cm}{ }^{-2}$ & $(1.0 \pm 0.2) \times 10^{17} \mathrm{~mol} \mathrm{~cm}^{-2}$ & - \\
\hline \multirow{2}{*}{ 26th Nov. } & $15.0 \pm 3.1 \mathrm{ppb}$ & - & - \\
& $(5.9 \pm 1.2) \times 10^{14} \mathrm{~mol} \mathrm{~cm}^{-2}$ & - & - \\
\hline \multirow{2}{*}{ 27th Nov. } & $9.9 \pm 1.2 \mathrm{ppb}$ & $2.9 \pm 0.7 \mathrm{ppm}$ & - \\
& $(4.3 \pm 0.5) \times 10^{14} \mathrm{~mol} \mathrm{~cm}^{-2}$ & $(1.2 \pm 0.3) \times 10^{17} \mathrm{~mol} \mathrm{~cm}^{-2}$ & - \\
\hline
\end{tabular}

Notes. For each specie it is indicated the mixing ratio $(\mathrm{ppm} / \mathrm{ppb})$ at an altitude of $90 \mathrm{~km}$ and the column density $\left(\mathrm{mol} \mathrm{cm}^{-2}\right)$ integrated above $90 \mathrm{~km}$.

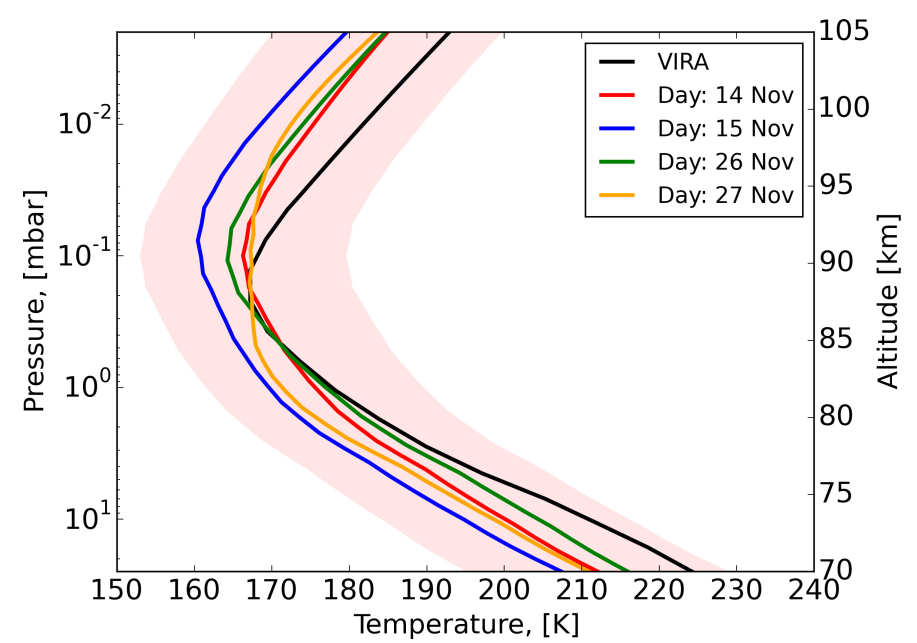

Fig. 11. Retrieved temperature profiles derived from the $\mathrm{CO}$ spectra integrated over the disk for the four days of observation: (red) November 14; (blue) November 15; (green) November 26; (orange) November 27. In black is plotted the VIRA temperature profile as reference. The colored area marks the uncertainty of the November 14 temperature profile.

ratio of $15.0 \pm 3.1 \mathrm{ppb}$ and one day later we observe a minimum value of $9.9 \pm 1.2 \mathrm{ppb} . \mathrm{H}_{2} \mathrm{O}$ appears to slightly decrease between November 14 and 27, ranging from $3.6 \pm 0.6 \mathrm{ppm}$ to $2.9 \pm 0.7 \mathrm{ppm} . \mathrm{SO}_{2}$ could be measured only on the first day of observation when we found a mixing ratio of $16.5 \pm 4.6 \mathrm{ppb}$.

\subsection{Mesospheric temperatures}

Four ALMA spectral maps provided a 3D picture of mesospheric temperatures $(\sim 70-105 \mathrm{~km})$ on the day side of Venus for both hemispheres. Figure 12 displays a map of ALMA vertical temperature retrievals for the first set of observation (November 14, 2011) compared to VIRA temperature profile. We present maps of vertical temperature retrievals for the other three days of observation in the Appendix A. Temperature profiles show at all latitudes and local times a similar behavior with a steady decrease of temperature up to an altitude of about $80-100 \mathrm{~km}$, followed by a regular increase of temperature. The altitude where the inversion occurs changes with local time: it occurs at $\sim 80 \mathrm{~km}$ in the morning (right side of the figure) and it increases toward the evening terminator (left side of the figure), where it occurs around $100 \mathrm{~km}$.
To study the variability of the thermal structure with latitude, local time and from one day to another, we plotted global temperature maps for the four sets of observation at two pressure levels: 11 mbar $(\sim 74 \mathrm{~km})$ and $0.01 \mathrm{mbar}(\sim 100 \mathrm{~km})$ (Fig. 13). At 11 mbar, temperatures increase by about $20 \mathrm{~K}$ from the morning side toward the evening terminator. A local maximum is observed in the southern hemisphere at high latitudes close to the evening terminator. Local minima are observed on the morning side. This trend is inverted at 0.01 mbar. A decrease of about $18 \mathrm{~K}$ occurs from the morning side to the evening terminator. The temperature field for the November 27 presents an exceptional behavior with a strong local maximum in the southern hemisphere near the morning side followed by a strong minimum on the northern hemisphere close to the evening terminator.

We analyzed the day-to-day variability by plotting the percentage difference between the thermal structure for the first day of observation and that of the other two days and the difference between the third and fourth day (see Fig. 14). As shown in Table 3 the total relative error on temperature is $8 \%$, therefore variations above this value represent real geophysical variabilities. The thermal structure does not seem to vary strongly from one day to another. Temperature on November 15 are colder compared to November 14. This behavior can already be seen in Fig. 13; however, as seen in Fig. 14, the percentage change from the first to the second day is less than $\pm 4-5 \%$, well within uncertainties on temperatures, considering our retrieval error $(8 \%)$ in Sect. 3. The difference in temperature between November 14 and 26 is even less pronounced. An exception is the thermal structure of the November 27. Compared to November 26 we observed percentage changes up to $\pm 12 \%$, well above the noise level, in particular above $90 \mathrm{~km}$.

\section{3. $S O$}

Figure 15 displays global maps of SO for the four days of observation. The SO spatial distribution varies strongly from one day to another. As for the disk-averaged retrievals, the SO abundance does not change much between November 14 and 15 . A maximum is then observed on November 26 followed by a strong decrease on November 27. The total error for the SO line changes between $9 \%$ and $21 \%$, therefore the variations observed are well above the noise level. Retrievals close to the limb need to be interpreted cautiously since our radiative transfer calculations do not include limb-sounding geometries. Each SO map displays also strong latitudinal and local time variations. On November 14, SO is present everywhere over the disk except on the night side. It seems enhanced in the northern hemisphere 


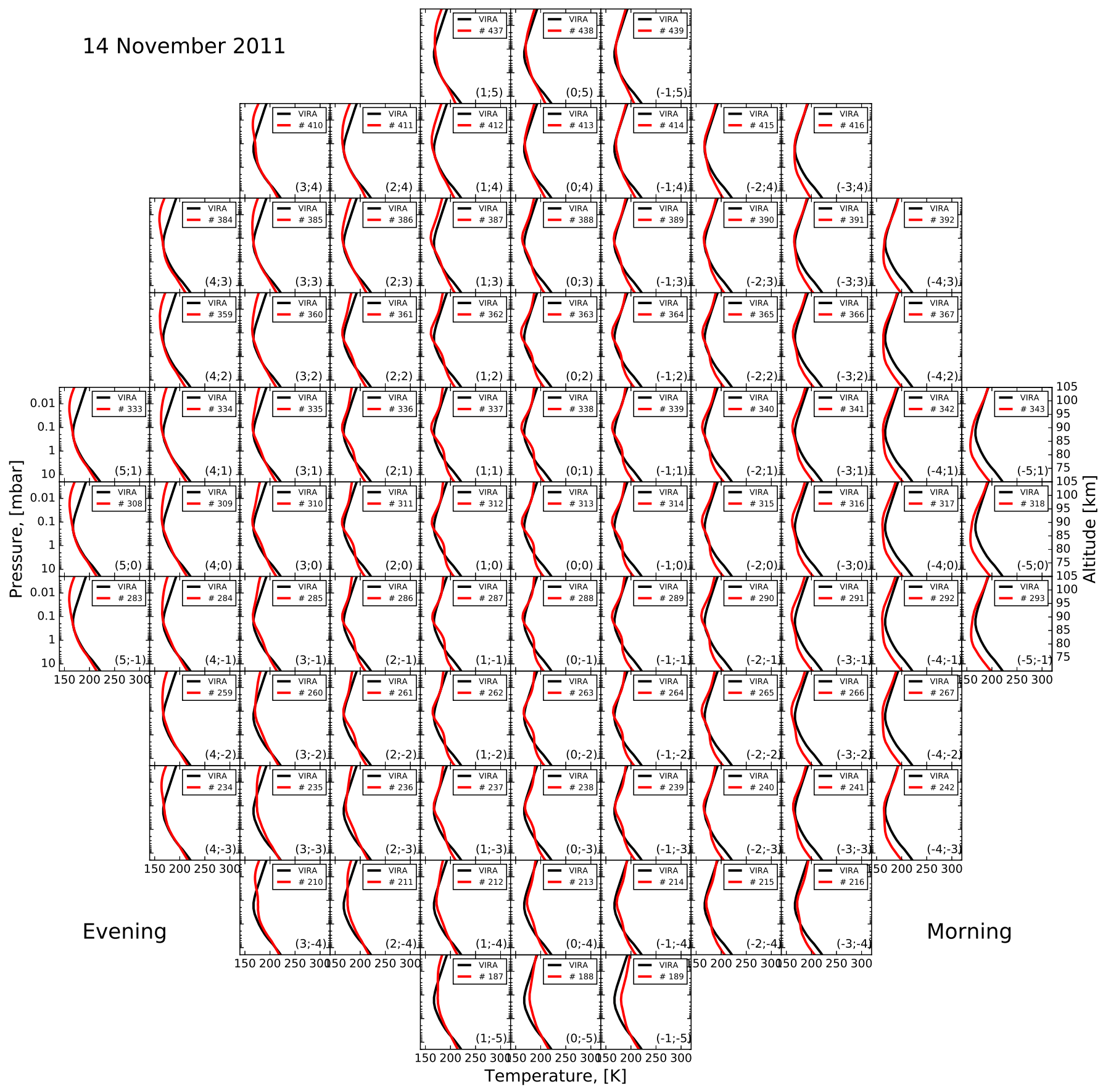

Fig. 12. Map of temperature retrievals (red lines) as function of pressure for the November 14, 2011. The black line correspond to the VIRA temperature profile. The right limb corresponds to the morning at local time $9 \mathrm{~h}$, and the left limb corresponds to the evening at local time $19 \mathrm{~h}$. The altitude scale on the right is approximate. Numbers in parentheses are the angular offsets relative to the planet center $(\Delta X, \Delta Y$ in $\operatorname{arcsec})$.

on the morning side and in the south near the evening terminator where a maximum is observed. A similar behavior is observed on November 26. On November 15, SO is enhanced uniformly near the evening terminator. November 27 exhibits a depletion of SO everywhere over the disk, except in the south near the evening terminator.

\subsection{Water}

Figure 16 displays global maps of $\mathrm{H}_{2} \mathrm{O}$ column density $\left(10^{17} \mathrm{~mol} \mathrm{~cm}^{-2}\right)$ for the first two sets of observation. The $\mathrm{S} / \mathrm{N}$ on November 26, and 27 is not sufficiently high to allow a retrieval of the $\mathrm{H}_{2} \mathrm{O}$ profiles. The HDO line is detected everywhere on the disk, for both days. Retrievals close to the limb need to be interpreted cautiously since our radiative transfer calculations do not include limb-sounding geometries. On November $14, \mathrm{H}_{2} \mathrm{O}$ abundance presents a minimum $\left(\sim 1 \times 10^{17} \mathrm{~mol} \mathrm{~cm}^{-2}\right)$ on the morning side, it increases with local time reaching a maximum value of $\sim 1.4 \times 10^{17} \mathrm{~mol} \mathrm{~cm}{ }^{-2} . \mathrm{H}_{2} \mathrm{O}$ exhibits a strong depletion by a factor of almost two from the first to the second day. This variation is above the noise level $( \pm 19 \%)$. Consequently, the observed abundance variability indicates real variations. On November 15 two local maxima with values of $\sim 0.5-1 \times 10^{17} \mathrm{~mol} \mathrm{~cm}{ }^{-2}$ were observed close the evening terminator in the northern hemisphere and at mid-latitudes in the southern hemisphere. 


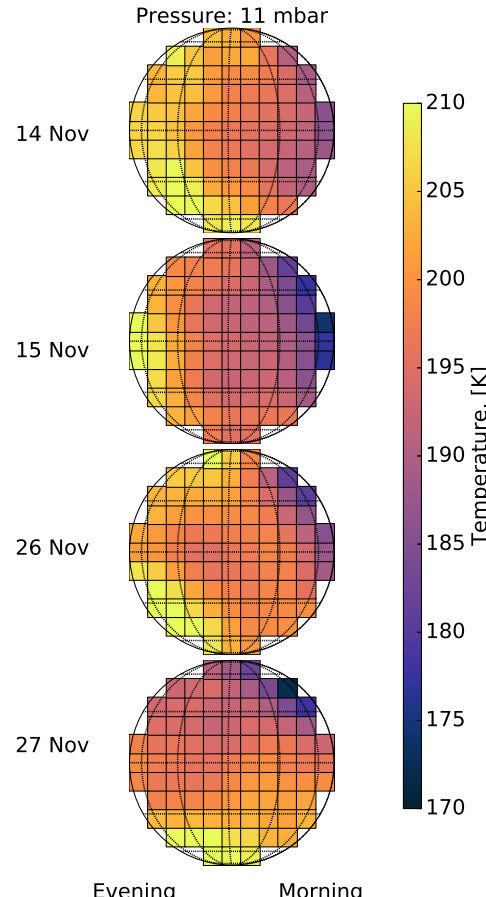

Evening

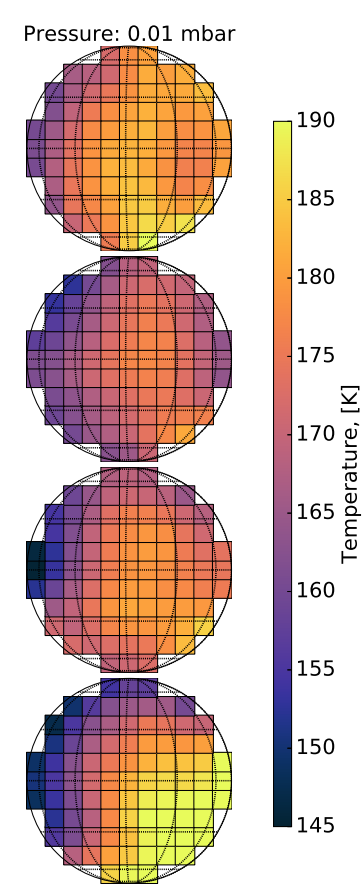

Evening
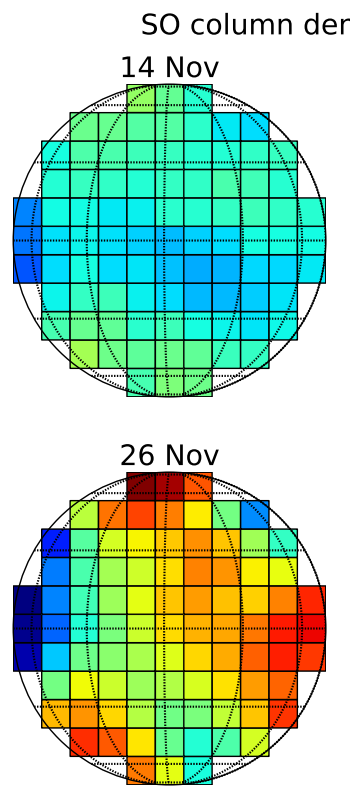

Evening
Morning

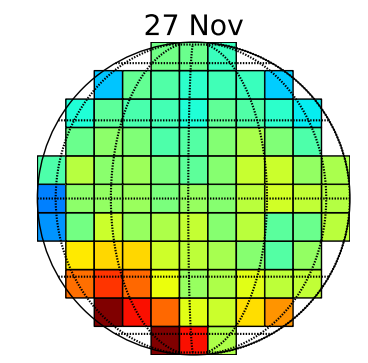

Morning

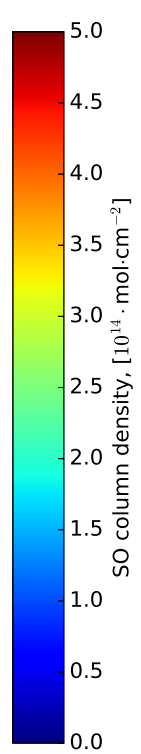

Fig. 15. Global maps of the retrieved ALMA SO column density $\left(10^{14} \mathrm{~mol} \mathrm{~cm}^{-2}\right)$ for the four days of observation.

\section{$\mathrm{H}_{2} \mathrm{O}$ column density, $\left[10^{17} \cdot \mathrm{mol} \cdot \mathrm{cm}^{-2}\right]$}

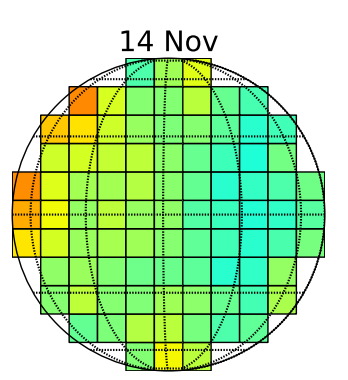

Evening

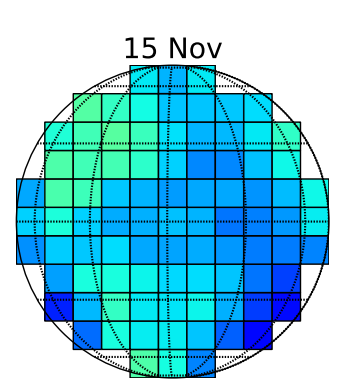

Morning

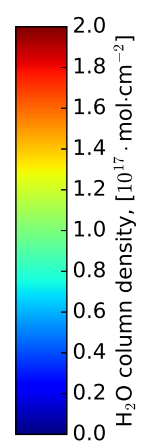

Fig. 16. Global maps of the retrieved ALMA $\mathrm{H}_{2} \mathrm{O}$ column density $\left(10^{17} \mathrm{~mol} \mathrm{~cm}^{-2}\right)$ for the first two days of observation.

able to acquire instantaneous vertical temperature profiles on the day side but their upper limit is $90 \mathrm{~km}$ (Tellmann et al. 2009). Ground-based observations, on the other hand, have a sparse temporal coverage. ALMA observations therefore allow to better constrain the day side of Venus upper mesosphere by providing three-dimensional maps of temperature.

We selected six examples of ALMA vertical temperature retrievals for the four sets of observation at different locations and compared them to previous measurements, both from spacecraft and ground-based observations (Fig. 17). We note that profiles obtained with different measurement techniques have different spatial resolutions, field of views and are obtained at different times. Here, we prefer to focus on latitudinal and local time variations, hence, we compare ALMA temperature profiles to the average profiles of each dataset.

In each panel of Fig. 17 we displayed the observation id number and within parentheses the angular offsets relative to the planet center $(\Delta X, \Delta Y$ in arcsec). Observations with right ascension $\Delta Y=0^{\prime \prime}$ are close to the equator, observations with $\Delta Y= \pm 3^{\prime \prime}$ correspond to a latitude of $\pm 50^{\circ}$ (see Fig. 12 for details). ALMA observations \#384, \#309, \#234 (see Fig. 12 for the locations of observations) are close to the evening terminator;

The majority of data acquired from experiments on board Venus Express cover the night side and the terminators of the planet. The radio-occultation experiment VeRa/VEx is the only one 


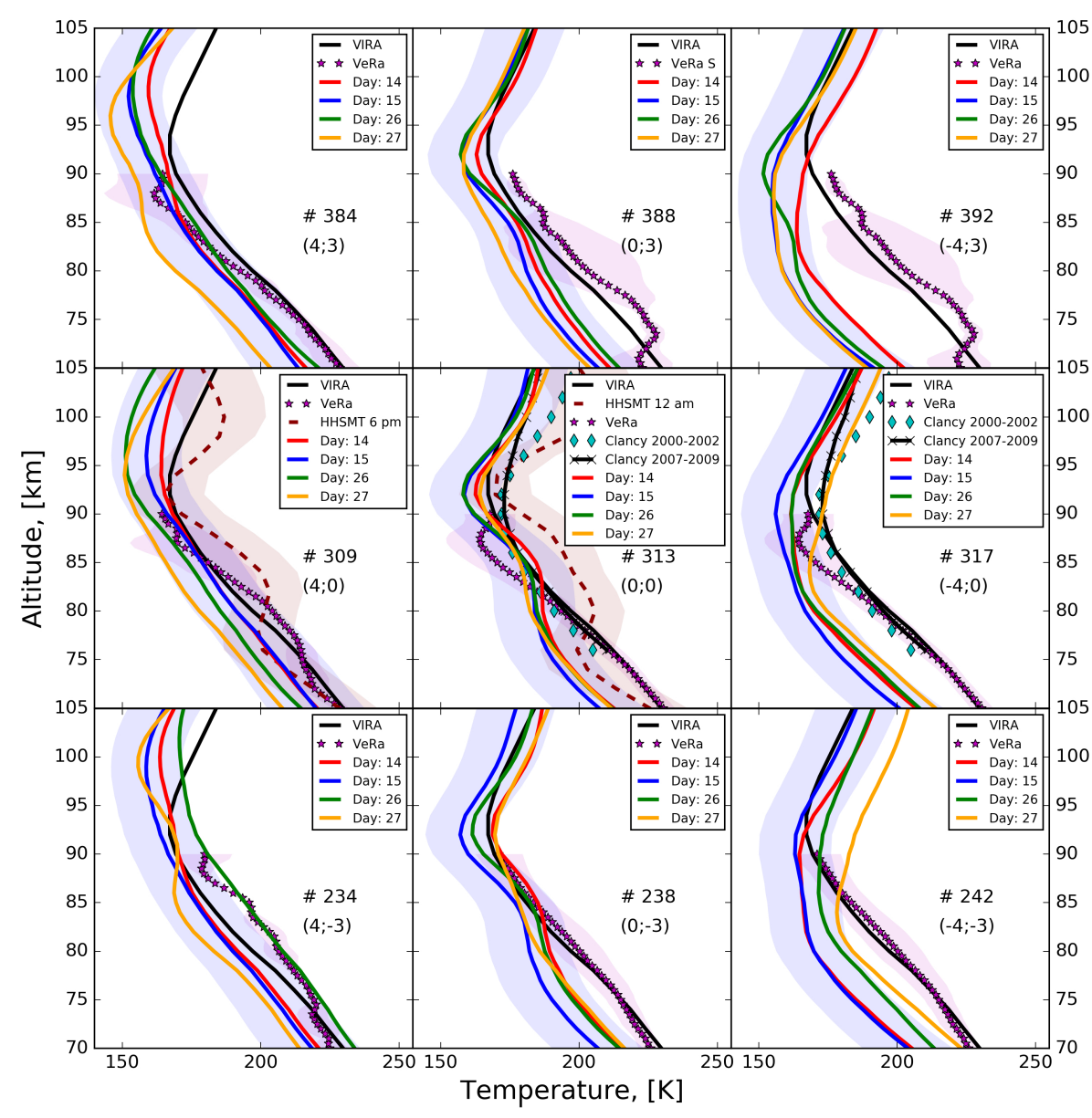

Fig. 17. ALMA vertical temperature profiles for the four days of observation at six different locations and local times (see Fig. 12). The VIRA (Seiff et al. 1985) models is indicated by solid black line. Vertical temperature profiles acquired by HHSMT ground-based measurements (purple dashed line) (Rengel et al. 2008a) and by the radio-occultation experiment $\mathrm{VeRa} / \mathrm{VEx}$ (magenta star symbols) are shown (Tellmann et al. 2009). Sub-mm groundbased observations are also displayed: cyan diamond (2000-2002 profile) and star symbols (2007-2009 profile) (Clancy et al. 2012). The colored area marks the uncertainty of temperature profiles. observations \#388, \#313, \#238 have a local time of approximately $13 \mathrm{~h}$; and observations \#392, \#317, \#242 have local time of approximately $10 \mathrm{~h}$.

For all latitudes and local times ALMA temperatures at altitudes $<90 \mathrm{~km}$ appear colder compared to the VIRA model (Seiff et al. 1985), used as a priori profile, and to the radiooccultation experiment VeRa/VEx profiles (Tellmann et al. 2009). This discrepancy increases toward the morning terminator. The VIRA thermal structure model was compiled based on the Venera and Pioneer Venus probes measurements and it is averaged over a latitudinal bin of $0-30^{\circ}$ and all local times. VeRa temperature profiles were averaged over latitude bins $0-30^{\circ}$ and $30-50^{\circ}$, assuming the symmetry of the northern and southern hemispheres, and over the local time bin 07-17 h. Discrepancies may be partially explained by temporal variability.

We then compare ALMA temperatures to those obtained by the Heinrich Hertz Submillimeter Telescope (HHSMT) groundbased observations (Rengel et al. 2008a,b). The HHSMT thermal structure is derived from ${ }^{12} \mathrm{CO} J=2-1$ at $230.54 \mathrm{GHz}$ at seven positions on the Venus disc and ${ }^{13} \mathrm{CO} J=2-1$ at $220.4 \mathrm{GHz}$ at one position. The FWHM (full width at half maximum) beam is detailed in Rengel et al. (2008a) and the angular diameter of Venus at the time of observations was 23.44". HHSMT profiles shown in Fig. 17 were derived by averaging all profiles corresponding to latitude $0^{\circ}$ and local time respectively of $18 \mathrm{~h}$ and $12 \mathrm{~h}$. Comparison with the ground-based observations obtained with HHSMT is good at the disk center for the local time $13 \mathrm{~h}$ below $75 \mathrm{~km}$. Above this altitude both ALMA and HHSMT reveal a complex thermal structure with alternating warmer and cooler layers rather than a constant increase or decrease of temperature. We detected a first inversion of the temperature at $\sim 80 \mathrm{~km}$ particularly visible at the disk center. A similar inversion is observed by HHSMT at a slightly lower altitude, however its temperature peaks at a much higher value than ALMA retrievals. At $\sim 90 \mathrm{~km}$ both ALMA and HHSMT observe a minimum in the temperature profiles followed by an increase. A similar behavior is observed also near the evening terminator $\left(\Delta X=4^{\prime \prime}\right)$. At this local time, both ALMA and HHSTM temperatures present a steady decrease up to a minimun around 90-100 km of altitude, followed by an increase.

Comparison with temperature profiles obtained by the James Clark Maxwell Telescope (JCMT) is possible only close to the equator on day side (Clancy et al. 2012). The JCMT temperature profiles are retrieved from the ${ }^{12} \mathrm{CO}$ at $345 \mathrm{GHz}$ and ${ }^{13} \mathrm{CO}$ at $330 \mathrm{GHz}$ line absorptions. Both the 2000-2002 and 2007-2009 profiles decrease with altitude reaching a minimum value around $90 \mathrm{~km}$ similar to ALMA temperatures. At the disk center there is a better agreement, in particular with the 2000-2002 profile, however JCMT does not observe the vertical inversion detected by ALMA between $80-90 \mathrm{~km}$. On the morning side $\left(\Delta X=-4^{\prime \prime}\right)$ ALMA and JCMT profiles are in good agreement above $90 \mathrm{~km}$, below this altitude ALMA temperature appears colder.

In conclusion, near the evening terminator and at $13 \mathrm{~h}$ there is a general agreement between different measurements. On the morning side, especially in the northern hemisphere, discrepancies between individual experiments are larger than uncertainties. These differences could be partly explained by a real variation of the thermal structure. Although limited to the night side, variations of approximately $10 \mathrm{~K}$ on timescales of $24 \mathrm{~h}$ up to $50 \mathrm{~K}$ on timescales of few (Earth) months were observed by previous experiments (Clancy et al. 2012; Mahieux et al. 2015; Piccialli et al. 2015). 


\subsection{Sulfur species}

Sulfur species $\left(\mathrm{SO}, \mathrm{SO}_{2}\right)$ in Venus mesosphere have been monitored since 2004 using ground-based observations in the submillimeter and in the IR (Sandor et al. 2010; Encrenaz et al. 2012, 2013, 2015, 2016). IR observations probe the cloud top at an altitude of $\sim 64 \mathrm{~km}$ and few kilometers below within the clouds (Encrenaz et al. 2016). Submillimeter measurements are sensitive to the altitude range of 70-100 km. Between 2006 and 2015, several instruments on board the European Spacecraft Venus Express and ground-based campaigns extensively investigated the dynamics and photochemistry of Venus mesosphere. Several studies reported large latitudinal and temporal variations of $\mathrm{SO}_{2}$, especially on long-term timescales (Marcq et al. 2013; Encrenaz et al. 2016; Sandor et al. 2010). Submillimeter observations showed evidence of highly variable $\mathrm{SO}$ abundance $(0.7$ to $31 \mathrm{ppb}$ ) from a day-to-day to one to two month timescales (Sandor et al. 2010). They also observed a strong depletion of $\mathrm{SO}$ and $\mathrm{SO}_{2}$ below $84 \mathrm{~km}$.

As seen in Fig. 9, ALMA SO spectra are mainly sensitive to the altitude region $80-100 \mathrm{~km}$ with a peak sensitivity around $92 \mathrm{~km}$. The same is valid for $\mathrm{SO}_{2}$. Both $\mathrm{SO}$ and $\mathrm{SO}_{2}$ retrievals exhibit a negligible abundance over $80-85 \mathrm{~km}$ followed by an increase with altitude in the mesosphere. This behavior is consistent with previous observations (Sandor et al. 2010). However, the altitude boundary between negligible SO (below), and significant SO (above) cannot be precisely determined due to a low $\mathrm{S} / \mathrm{N}$.

From disk-averaged spectra, we found the SO abundance at $90 \mathrm{~km}$ to highly vary from one day to another. We observe in particular a depletion of SO between November 26 and 27 with the SO abundance decreasing from $15 \pm 3.1 \mathrm{ppb}$ to $9.9 \pm 1.2 \mathrm{ppb}$ (Table 4). Since at the time of our observations the illumination fraction was $92 \%$, these measurements refer to the day side. They lie well within the values reported by Sandor et al. (2010). Due to a low $\mathrm{S} / \mathrm{N}, \mathrm{SO}_{2}$ could be retrieved from a disk-averaged spectrum only on the first day of observation when we found an abundance of $16.5 \pm 4.6 \mathrm{ppb}$. The ratio $\mathrm{SO}_{2} / \mathrm{SO}$ is $1.5 \pm 0.4$, in good agreement with the mean daytime ratio of $1.9 \pm 1.2$ derived by Sandor et al. (2010).

A valuable capability of ALMA is to provide high resolution maps of the Venus disk at a given time and therefore, to study latitude/local time variations as well as temporal variations from one day to another. SO abundance at $90 \mathrm{~km}$ varies between $<1$ and $15 \mathrm{ppb}\left(<1-5 \times 10^{14} \mathrm{~mol} \mathrm{~cm}^{-2}\right)$ over the four sets of observation. Global maps of ALMA SO abundances reveal strong variations both with latitude and local time on each day and global variations on a day-to-day timescale. As reported by Sandor et al. (2010), the physical and chemical mechanisms that produce variations of $\mathrm{SO}$ and $\mathrm{SO}_{2}$ are still unknown. Both altitude and temporal variability of SO imply the existence of an additional reservoir in the upper mesosphere (Sandor et al. 2010; Jessup et al. 2015). Sandor et al. (2010) suggests, moreover, a possible link between temporal variations of SO and those of water vapor and $\mathrm{CO}$.

\subsection{Water}

Ground-based observations of HDO at submillimeter wavelengths showed a strong variability in the mesosphere: Sandor \& Clancy (2005) studied a set of long-term measurements (1998-2004) of water vapor in Venus' mesososphere. They found a strong global variability with $\mathrm{H}_{2} \mathrm{O}$ ranging from $<0.1$ to $3.5 \mathrm{ppmv}$ on a short (one to two month) timescale.
These results were later confirmed by a study from Gurwell et al. (2007) who detected a decrease in the water abundance by a factor of nearly 50 in less than $48 \mathrm{hr}$. SOIR/Venus Express simultaneously retrieved vertical profiles of $\mathrm{HDO}$ and $\mathrm{H}_{2} \mathrm{O}$ in the Venus mesosphere at the terminator and for different latitudes finding temporal variations for the mixing ratios of HDO and $\mathrm{H}_{2} \mathrm{O}$ not exceeding two to three times the mean value (Fedorova et al. 2008). More recently, Fedorova et al. (2016) analyzed SPICAV/VEx measurements of $\mathrm{H}_{2} \mathrm{O}$ abundance above Venus' clouds $(60-62 \mathrm{~km})$ acquired during the eight years of Venus Express operations. They suggest that short timescales variations of $\mathrm{H}_{2} \mathrm{O}$ could be explained as a real variation of the mixing ratio and/or possible variations of the cloud opacity within the clouds.

While Sandor \& Clancy (2005) could not identify any clear diurnal pattern, probably masked by the one to two month variability, Gurwell et al. (2007) found some indication of higher water vapor abundances just before dawn (local time $\sim 5 \mathrm{~h}$ ). A minimum in the water vapor abundance seemed to occur around $11 \mathrm{~h}$, followed by an increase during the day, however the number of measurements was not enough to confirm a clear pattern. Gurwell et al. (2007) also suggested as possible mechanism for the rapid variability of water vapor a moderate variation of temperature of about 10-15 K. Venus' mesosphere is dominated by large density and temperature variations (Clancy et al. 2012; Mahieux et al. 2015; Piccialli et al. 2015), which could be the cause of the water vapor variability.

ALMA HDO spectra are sensitive to altitudes $80-100 \mathrm{~km}$ with a peak sensitivity around $\sim 94 \mathrm{~km}$ (See Fig. 10). Best-fit $\mathrm{H}_{2} \mathrm{O}$ profiles, inferred from HDO, are characterized by a negligible abundance $(<0.1 \mathrm{ppm})$ below $\sim 82 \mathrm{~km}$ altitudes. However, the altitude boundary between negligible $\mathrm{H}_{2} \mathrm{O}$ (below), and significant $\mathrm{H}_{2} \mathrm{O}$ (above) cannot be precisely determined due to a low S/N. Sandor \& Clancy (2005) also measured values of $\mathrm{H}_{2} \mathrm{O}$ less than $0.1 \mathrm{ppmv}$ in the altitude range $65-100 \mathrm{~km}$ on different dates. Moreover, a marked depletion of $\mathrm{H}_{2} \mathrm{O}$ and $\mathrm{HDO}$ is seen in SOIR/VEx solar occultation observations in the range $80-90 \mathrm{~km}$ at the terminator, as reported by Bertaux et al. (2007), Fedorova et al. (2008). This trend is also confirmed by a detailed analysis of the whole SOIR dataset (Chamberlain et al. 2015). Fedorova et al. (2008) explains that the depletion in the mixing ratio near $85 \mathrm{~km}$ could be related to the depletion in the $\mathrm{CO}_{2}$ density near $95 \mathrm{~km}$ and to a possible temperature inversion at these altitudes, however no clear conclusion could be drawn. ALMA disk-averaged $\mathrm{H}_{2} \mathrm{O}$ abundances presents limited temporal variations ranging from $3.6 \pm 0.6 \mathrm{ppm}$ on the 14 th to $2.9 \pm 0.7 \mathrm{ppm}$ on the November 27 (see Table 4). These values are within the range of previous results (Gurwell et al. 2007).

As for $\mathrm{SO}$, we retrieved $\mathrm{H}_{2} \mathrm{O}$ maps: they show a decrease of water vapor from November 15 to 15 (Fig. 16). As suggested by Gurwell et al. (2007), such variation may be correlated to a decrease of temperatures all over Venus' disk of about 5-9 K from the first to the second day of measurements observed at 0.01 mbar (See Fig. 14). However, as remarked previously, these temperature variations lie within the uncertainty on temperature $(8 \%)$, consequently it is hard to draw a definite conclusion.

On both days a diurnal pattern can be identified, with an increase of water vapor abundance over the day (Fig. 16). Such variations cannot be correlated to the temperature field at $0.01 \mathrm{mbar}(100 \mathrm{~km})$, in fact at this altitude temperatures decrease from the morning side toward the evening. Sandor et al. (2010) proposes a possible link between temporal variations of SO and water vapor. Nevertheless, as for SO, the driving mechanisms for water vapor variability are still unknown. 


\section{Summary and conclusions}

The thermal structure and minor species of Venus mesosphere (70-105 km) were observed with the Atacama Large Millimeter Array (ALMA) at four different dates in November 2011. At the time of observations the illumination factor was $92 \%$, so that most of the measurements were acquired on the day side. The thermal structure of Venus upper mesosphere on the day side is poorly known, ALMA observations are thus extremely valuable to constrain this atmospheric region.

We inverted 3D maps of the mesospheric thermal structure from the $\mathrm{CO}$ line at $345.795 \mathrm{GHz}$. The main features observed in the temperature profiles are: (i) a constant decrease of temperature up to $80-100 \mathrm{~km}$, depending on the local time; (ii) a steady decrease of temperature above $80-100 \mathrm{~km}$; and (iii) the altitude where this inversion occurs increases from $80 \mathrm{~km}$ in the morning to about $100 \mathrm{~km}$ near the evening terminator. Global maps of temperature at $11 \mathrm{mbar}(\sim 74 \mathrm{~km})$ present an increase from the morning toward the evening terminator. This trend is inverted at $0.01 \mathrm{mbar}(\sim 100 \mathrm{~km})$. Global temperature fields do not reveal strong day-to-day variability, an exception is the thermal structure of the November 27 at 0.01 mbar with a strong maximum localized in the southern hemisphere on the morning followed by a minimum on the north hemisphere toward the evening terminator.

Temperature profiles were then used to retrieve the $\mathrm{SO}, \mathrm{SO}_{2}$, and $\mathrm{H}_{2} \mathrm{O}$ abundances. We used $\mathrm{HDO}$ as a tracer for water assuming a $\mathrm{D} / \mathrm{H}$ enrichment of 200 times the terrestrial value. SO, $\mathrm{SO}_{2}$, and $\mathrm{H}_{2} \mathrm{O}$ are characterized by a negligible abundance below $\sim 85 \mathrm{~km}$ followed by an increase with altitude in the upper mesosphere. Due to a low $\mathrm{S} / \mathrm{N}$, however, the altitude boundary between the depleted (below) and the enriched (above) layer cannot be determined precisely. Disk-averaged SO abundances do not show high temporal variability. An exception is November 26 when a maximum mixing ratio of $15.0 \pm 3.1 \mathrm{ppb}$ is followed the next day by a minimum value of $9.9 \pm 1.2 \mathrm{ppb}$. Due to a very low $\mathrm{S} / \mathrm{N}, \mathrm{SO}_{2}$ could only be derived from the disk-averaged spectrum on the first day of observation revealing an abundance of $16.5 \pm 4.6 \mathrm{ppb}$. We found a $\mathrm{SO}_{2} / \mathrm{SO}$ ratio of $1.5 \pm 0.4$, in good agreement with the mean daytime ratio of $1.9 \pm 1.2$ derived by Sandor et al. (2010). $\mathrm{H}_{2} \mathrm{O}$ disk-averages retrievals reveal a steady decrease from November 14 to 27, with the abundance varying from $3.6 \pm 0.6 \mathrm{ppm}$ on the first day to $2.9 \pm 0.7 \mathrm{ppm}$ on the last day.

Thanks to the imaging capabilities of ALMA, it was possible for the first time to acquire instantaneous maps of SO and water vapor. Global maps of SO abundance at $90 \mathrm{~km}$ reveal a strong variability both with latitude/local time and on a global scale from one day to another. The SO abundance varies between $<1$ and $15 \mathrm{ppb}$, depending on the location and the day. We were able to retrieve maps of $\mathrm{H}_{2} \mathrm{O}$ only for the first two runs of observation, due to a low $\mathrm{S} / \mathrm{N}$. On each day, $\mathrm{H}_{2} \mathrm{O}$ is almost uniform all over Venus' disk. The driving mechanisms for the variability of both SO and water abundance are still unknown. Sandor et al. (2010) suggested a possible link between temporal variations of SO and those of water vapor and CO. Gurwell et al. (2007) proposed that the water vapor variability may be partly correlated to variations in temperature. In this study we cannot draw any definite conclusion about these correlations due to a low S/N.

In conclusion, ALMA offers a unique opportunity to monitor the complex dynamics, temperature structure and chemistry of Venus upper atmosphere. In the future we plan new simultaneous observations with ALMA to characterize the thermal structure and $\mathrm{HDO}, \mathrm{H}_{2} \mathrm{O}$ abundances on both the day and night side and to investigate possible correlations.

Acknowledgements. This paper makes use of the following ALMA data: ADS/JAO.ALMA\#2011.0.00136.S. ALMA is a partnership of ESO (representing its member states), NSF (USA) and NINS (Japan), together with NRC (Canada), NSC and ASIAA (Taiwan), and KASI (Republic of Korea), in cooperation with the Republic of Chile. The Joint ALMA Observatory is operated by ESO, AUI/NRAO and NAOJ. We acknowledge support from the FP-7 project EUROVENUS of the European Union.

\section{References}

Belyaev, D. A., Montmessin, F., Bertaux, J.-L., et al. 2012, Icarus, 217, 740 Bertaux, J.-L., Vandaele, A.-C., Korablev, O., et al. 2007, Nature, 450, 646 Bézard, B., \& de Bergh, C. 2007, J. Geophys. Res. (Planets), 112, E04S07 Bézard, B., de Bergh, C., Crisp, D., \& Maillard, J.-P. 1990, Nature, 345, 508 Bézard, B., Tsang, C. C. C., Carlson, R. W., et al. 2009, J. Geophys. Res. (Planets), 114, E00B39

Chamberlain, S., Wilquet, V., Mahieux, A., et al. 2015, in EGU General Assembly Conference Abstracts, 17, 8331

Clancy, R. T., Sandor, B. J., \& Moriarty-Schieven, G. H. 2003, Icarus, 161, 1 Clancy, R. T., Sandor, B. J., \& Moriarty-Schieven, G. 2012, Icarus, 217, 779

Conrath, B. J., Gierasch, P. J., \& Ustinov, E. A. 1998, Icarus, 135, 501

Encrenaz, T., Greathouse, T. K., Roe, H., et al. 2012, A\&A, 543, A153

Encrenaz, T., Greathouse, T. K., Richter, M. J., et al. 2013, A\&A, 559, A65

Encrenaz, T., Moreno, R., Moullet, A., Lellouch, E., \& Fouchet, T. 2015, Planet. Space Sci., 113, 275

Encrenaz, T., Greathouse, T. K., Richter, M. J., et al. 2016, A\&A, 595, A74

Fedorova, A., Korablev, O., Vandaele, A.-C., et al. 2008, J. Geophys. Res., 113

Fedorova, A., Marcq, E., Luginin, M., et al. 2016, Icarus, 275, 143

Fouchet, T., Greathouse, T. K., Spiga, A., et al. 2016, Icarus, 277, 196

Guerlet, S., Fouchet, T., Bézard, B., Simon-Miller, A. A., \& Michael Flasar, F. 2009, Icarus, 203, 214

Gurwell, M. A., Melnick, G. J., Tolls, V., Bergin, E. A., \& Patten, B. M. 2007, Icarus, 188, 288

Ho, W., Kaufman, I. A., \& Thaddeus, P. 1966, J. Geophys. Res., 71, 5091

Jessup, K. L., Marcq, E., Mills, F., et al. 2015, Icarus, 258, 309

Lellouch, E., \& Witasse, O. 2008, Planet. Space Sci., 56, 1317

Lellouch, E., Paubert, G., Moreno, R., \& Moullet, A. 2008, Planet. Space Sci., 56,1355

Lellouch, E., Gurwell, M., Butler, B., et al. 2017, Icarus, 286, 289

Mahieux, A., Vandaele, A., Bougher, S., et al. 2015, Planet. Space Sci., 113, 309

Marcq, E., Bézard, B., Drossart, P., et al. 2008, J. Geophys. Res. (Planets), 113

Marcq, E., Belyaev, D., Montmessin, F., et al. 2011, Icarus, 211, 58

Marcq, E., Bertaux, J.-L., Montmessin, F., \& Belyaev, D. 2013, Nature Geoscience, 6, 25

Mills, F., Esposito, L. W., \& Yung, Y. K. 2007, in Exploring Venus as terrestrial planet, Geophys. Monograph Ser., 176, 73

Piccialli, A., Montmessin, F., Belyaev, D., et al. 2015, Planet. Space Sci., 113, 321

Pickett, H. M., Poynter, R. L., Cohen, E. A., et al. 1998, J. Quant. Spectr. Rad. Transf., 60, 883

Poynter, R. L., \& Pickett, H. M. 1985, Appl. Opt., 24, 2235

Rengel, M., Hartogh, P., \& Jarchow, C. 2008a, Planet. Space Sci., 56, 1688

Rengel, M., Hartogh, P., \& Jarchow, C. 2008b, Planet. Space Sci., 56, 1368

Rothman, L. S., Jacquemart, D., Barbe, A., et al. 2005, J. Quant. Spectr. Rad. Transf., 96, 139

Sandor, B. J., \& Clancy, R. T. 2005, Icarus, 177, 129

Sandor, B. J., Todd Clancy, R., Moriarty-Schieven, G., \& Mills, F. P. 2010, Icarus, 208, 49

Sandor, B. J., Clancy, R. T., \& Moriarty-Schieven, G. 2012, Icarus, 217, 839

Seiff, A., Schofield, J. T., Kliore, A. J., Taylor, F. W., \& Limaye, S. S. 1985, Adv. Space Res., 5, 3

Sonnabend, G., Krötz, P., Schmülling, F., et al. 2012, Icarus, 217, 856

Tellmann, S., Pätzold, M., Häusler, B., Bird, M. K., \& Tyler, G. L. 2009, J. Geophys. Res. (Planets), 114, E00B36

Varanasi, P. 1971, J. Quant. Spectr. Rad. Transf., 11, 223

Varanasi, P., Chudamani, S., \& Kapur, S. 1987, J. Quant. Spectr. Rad. Transf., 38,167

Zhang, X., Liang, M. C., Mills, F. P., Belyaev, D. A., \& Yung, Y. L. 2012, Icarus, 217,714 


\section{Appendix A: Temperature maps}

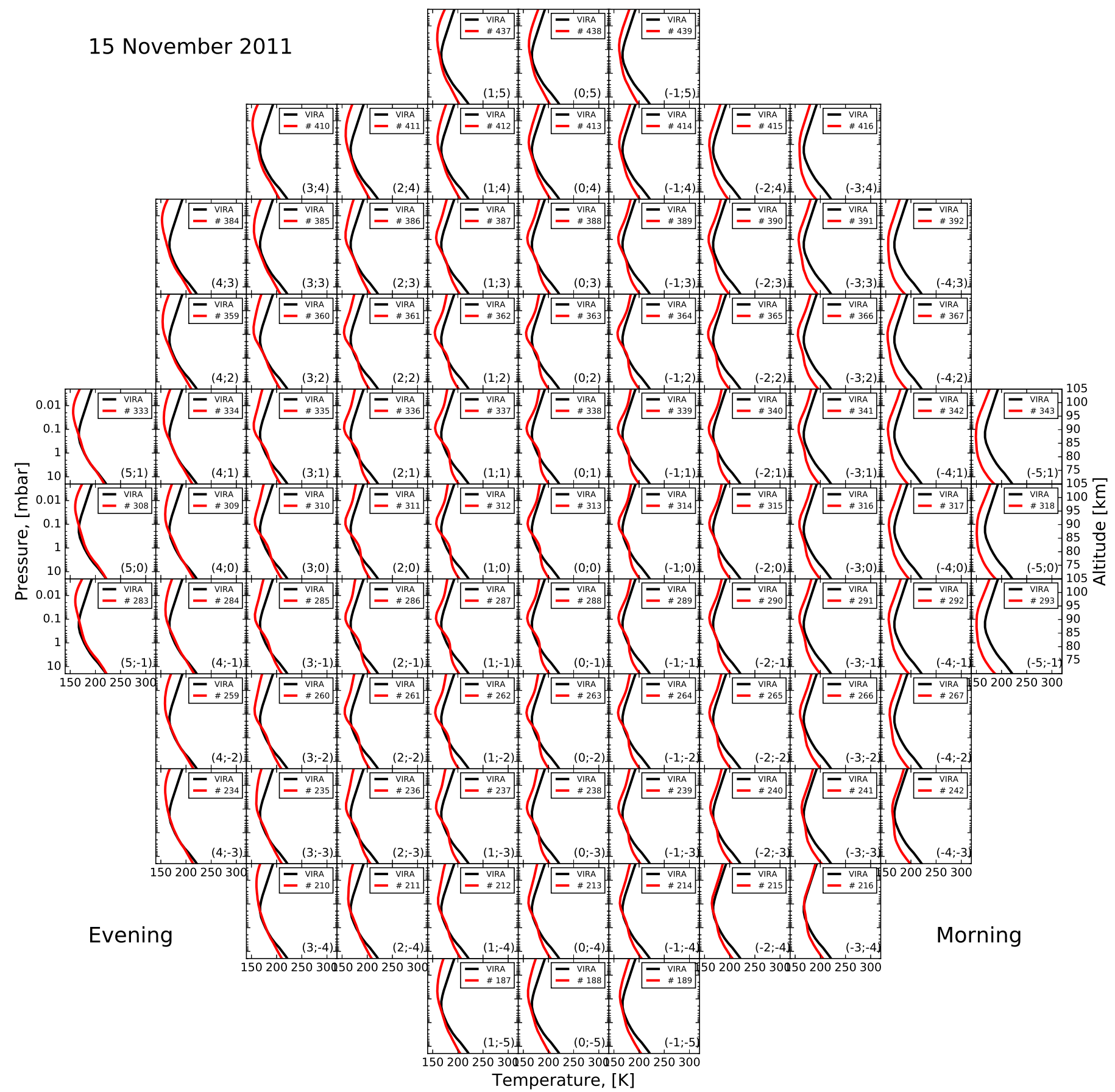

Fig. A.1. Map of temperature retrievals (red lines) as function of pressure for November 15, 2011. The black line correspond to the VIRA temperature profile. The right limb corresponds to the morning at local time $9 \mathrm{~h}$, and the left limb corresponds to the evening at local time $19 \mathrm{~h}$. The altitude scale on the right is approximate. Numbers in parentheses are the angular offsets relative to the planet center $(\Delta X, \Delta Y$ in $\operatorname{arcsec})$. 


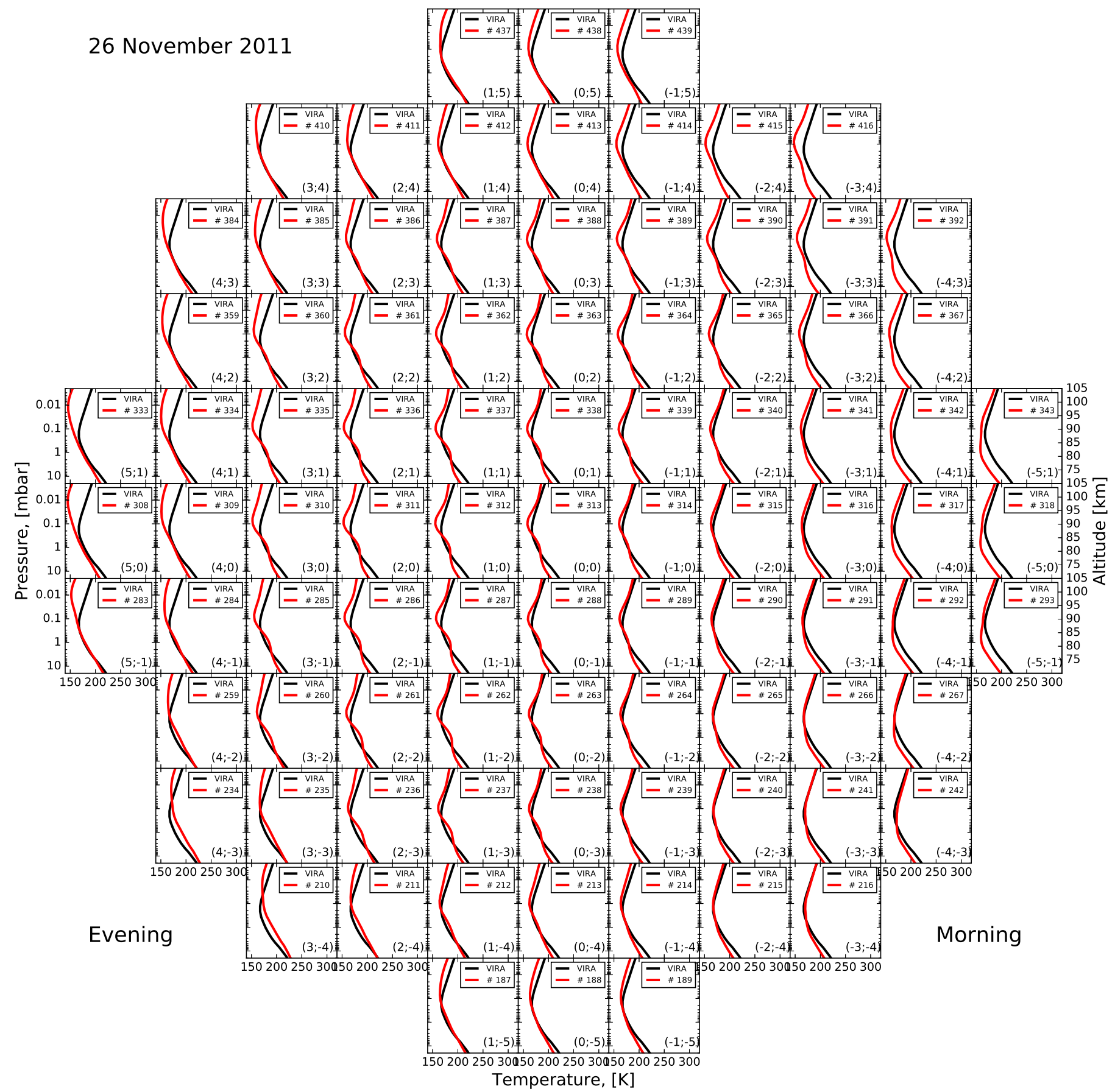

Fig. A.2. Map of temperature retrievals (red lines) as function of pressure for November 26, 2011. The black line correspond to the VIRA temperature profile. The right limb corresponds to the morning at local time $9 \mathrm{~h}$, and the left limb corresponds to the evening at local time $19 \mathrm{~h}$. The altitude scale on the right is approximate. Numbers in parentheses are the angular offsets relative to the planet center $(\Delta X, \Delta Y$ in $\operatorname{arcsec})$. 


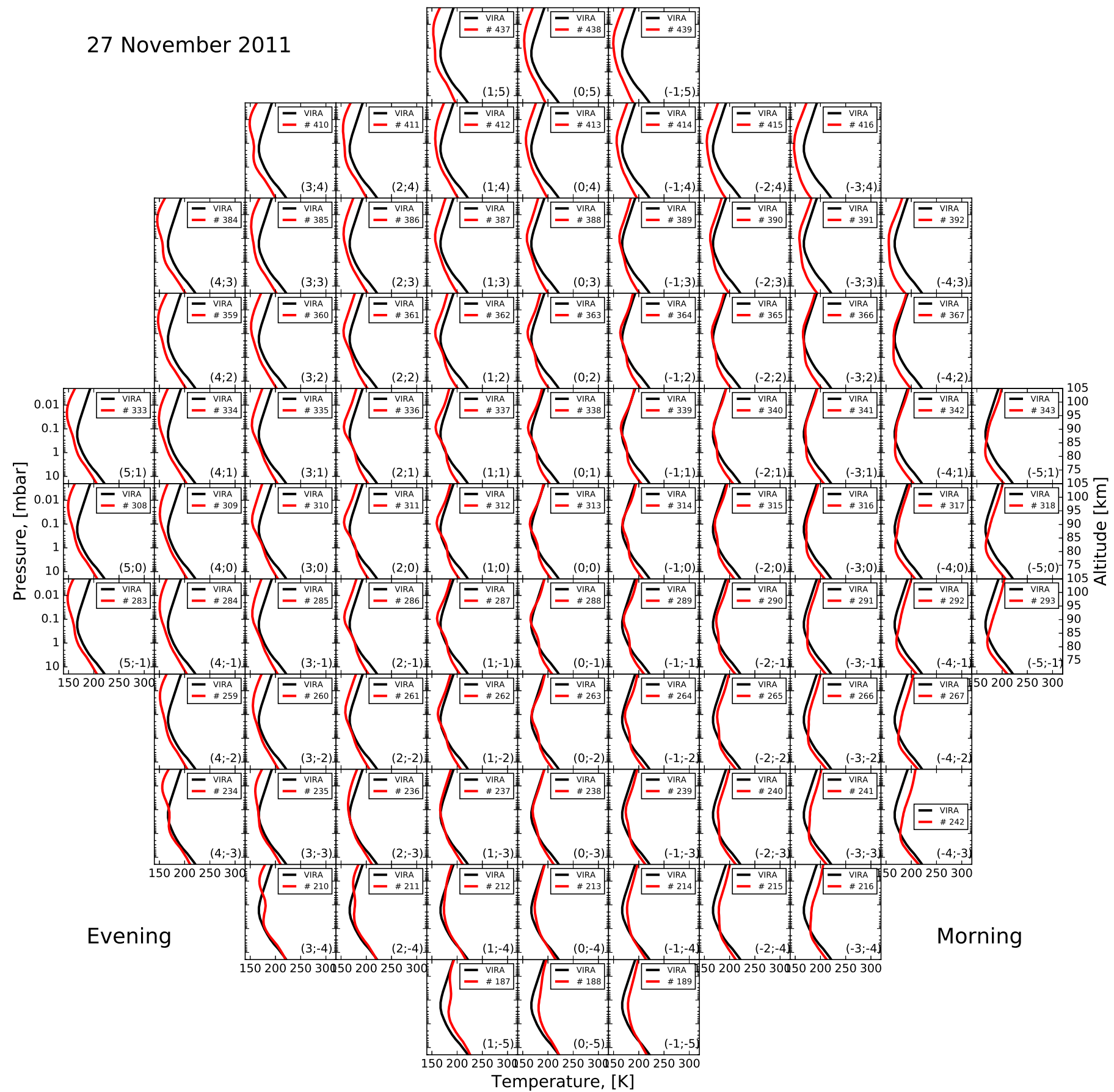

Fig. A.3. Map of temperature retrievals (red lines) as function of pressure for November 27, 2011. The black line correspond to the VIRA temperature profile. The right limb corresponds to the morning at local time $9 \mathrm{~h}$, and the left limb corresponds to the evening at local time $19 \mathrm{~h}$. The altitude scale on the right is approximate. Numbers in parentheses are the angular offsets relative to the planet center $(\Delta X, \Delta Y$ in $\operatorname{arcsec})$. 